\title{
HIGH-DIMENSIONAL HELICITIES AND RIGIDITY OF LINKED FOLIATIONS *
}

\author{
TRISTAN RIVIÈRE ${ }^{\dagger}$
}

\begin{abstract}
We give an ergodic interpretation of Hopf-Novikov helicities as conjectured by V.I Arnold in [1]. We then extend to higher dimension the topological lower bounds obtained by M.Freedman and Z.X. He in [8] for energies of invariant forms of linked foliations.
\end{abstract}

\section{Introduction.}

1.1. Arnold's ergodic interpretation of the generalized Hopf Invariant and rigidity of knotted magnetic tubes. In their paper [7] and [8] M.H. Freedman and Z.-X. He consider the following problem : Let $K$ be a knot in $\mathbb{R}^{3}$ (a regular embedding of $S^{1}$ in $\mathbb{R}^{3}$ ) and $T$ be a regular tubular neighborhood of $K$ in $\mathbb{R}^{3}$, one considers closed 2-forms $\omega$ in $\bar{T}$ such that the restriction of $\omega$ to the boundary of $T$ is 0 (i.e. $\iota_{\partial T}^{*} \omega=0$, where $\iota_{\partial T}$ is the inclusion map). They proved that for any such 2-form the following inequality holds

$$
\frac{1}{4 \pi} \int_{T} \int_{T} \frac{|\omega(x)||\omega(y)|}{|x-y|^{2}} \geq[\operatorname{Flux}(\omega)]^{2} \operatorname{ac}(K)
$$

where $\operatorname{Flux}(\omega)$ is the integral of $\omega$ over any surface in $\bar{T}$ whose boundary lies in $\partial T$ and whose intersection number with $K$ is +1 . Moreover $\operatorname{ac}(K)$ is the following knot invariant :

Let $L$ be an embedded oriented closed curve in $T$, we denote by $\operatorname{deg} L$ the intersection number of $L$ with any oriented section of $T$ generating $H_{2}(T, \partial T ; \mathbb{Z}$ ) (oriented such that the intersection number between $\Sigma$ and $K$ is +1$)$. Then

$$
\operatorname{ac}(K)=\inf \left\{\frac{\mathrm{c}\left(L, L^{\prime}\right)}{|\operatorname{deg} L|\left|\operatorname{deg} L^{\prime}\right|} \quad ; \quad L, L^{\prime} \begin{array}{l}
\text { closed emb. } \\
\text { curves in } T
\end{array}\right\}
$$

where c $\left(L, L^{\prime}\right)$ is the over-crossing number of $L$ and $L^{\prime}$ : the minimal number of overcrossing of $L$ over $L^{\prime}$ among all planar knot diagrams representing $\left(L, L^{\prime}\right)$. The result above is also extended to the case of a general link (see [8]).

Since $\omega$ is a closed 2-form in dimension 3 , in a neighborhood $U$ of a point $x_{0}$ such that $\omega\left(x_{0}\right) \neq 0$, using Darboux theorem, $\omega$ can be written as $\omega=\phi^{*}\left(d x_{1} \wedge d x_{2}\right)$, where $\phi: U \rightarrow D^{2}$.

In the very particular case where $\omega$ can be written globally as $\omega=\phi^{*}\left(d x_{1} \wedge d x_{2}\right)$ where $\phi: T \rightarrow D^{2}$, the proof of (1.1) can be sketched as follows : Using Federer's coarea formula we have

$$
\begin{gathered}
\frac{1}{4 \pi} \int_{T \times T}\left|X_{\phi}(x) \wedge X_{\phi}(y) \cdot \frac{x-y}{|x-y|^{3}}\right| d x d y \\
=\frac{1}{4 \pi} \int_{D^{2}} d \sigma(\xi) \int_{D^{2}} d \sigma(\zeta) \int_{\phi^{-1}(\xi) \times \phi^{-1}(\zeta)}\left|\vec{t}(x) \wedge \vec{t}(y) \cdot \frac{x-y}{|x-y|^{3}}\right|
\end{gathered}
$$

${ }^{*}$ Received June 18, 2002; accepted for publication July 12, 2002.

${ }^{\dagger}$ D-Math, ETH-Zentrum, 8092 Zürich, Switzerland (riviere@math.ethz.ch). 
where $X_{\phi}$ is the divergence free field associated to $\omega\left(\iota_{X_{\phi}} d x d y d z=\omega-\iota\right.$ is the interior product of vectors on forms - and this yields $X_{\phi} \in \operatorname{Ker}(\omega)$ and $\left.\left\langle * \omega ; X_{\phi}\right\rangle=|\omega|^{2}\right)$, $d \sigma$ is the volume form on $D^{2}, \vec{t}$ is the unit vector tangent to the preimages of regular points by $\phi$ and the measure on $\phi^{-1}(\xi) \times \phi^{-1}(\zeta)$ is the product measure obtained from the restriction of the ambiant metric of $\mathbb{R}^{3}$ to $\phi^{-1}(\xi)$ and $\phi^{-1}(\zeta)$. Consider two regular points of $\phi$ in $D^{2}, \xi$ and $\zeta$, for $(x, y) \in \phi^{-1}(\xi) \times \phi^{-1}(\zeta),\left|\vec{t}(x) \wedge \vec{t}(y) \cdot \frac{x-y}{|x-y|^{3}}\right|$ is the norm of the pull-back of the volume form on $S^{2}: d \mathrm{vol}_{S^{2}}$, by the map $L$

$$
\begin{gathered}
L \quad: \quad \phi^{-1}(\eta) \times \phi^{-1}(\xi) \longrightarrow S^{2} \\
(x, y) \longrightarrow \frac{x-y}{|x-y|}
\end{gathered}
$$

Thus we have

$$
\int_{\phi^{-1}(\xi) \times \phi^{-1}(\zeta)}\left|\vec{t}(x) \wedge \vec{t}(y) \cdot \frac{x-y}{|x-y|^{3}}\right|=\int_{s \in S^{2}} \operatorname{Card}\left(L^{-1}(s)\right) d \operatorname{vol}_{S^{2}}
$$

For any regular value $s$ of $L, \operatorname{Card}\left(L^{-1}(s)\right)$ is the number of over-crossing of $\phi^{-1}(\eta)$ over $\phi^{-1}(\xi)$ for the projection on an oriented plane orthogonal to $s$. Thus (1.4) combined with the definition of $\operatorname{ac}(K)$ implies:

$$
\frac{1}{4 \pi} \int_{\phi^{-1}(\xi) \times \phi^{-1}(\zeta)}\left|\vec{t}(x) \wedge \vec{t}(y) \cdot \frac{x-y}{|x-y|^{3}}\right| \geq \operatorname{ac}(K) \operatorname{deg} \phi^{-1}(\xi) \operatorname{deg} \phi^{-1}(\eta)
$$

Using once again the coarea formula, we have, for any generator $\Sigma$ of $H_{2}(T, \partial T, \mathbb{Z})$ whose intersection number with $K$ is +1 ,

$$
\operatorname{Flux}\left(\phi^{*} d \sigma\right)=\int_{\Sigma} \phi^{*} d \sigma=\int_{D^{2}} d \sigma(\xi) \operatorname{deg} \phi^{-1}(\xi)
$$

Combining (1.3), (1.5) and (1.6) we establish (1.1) for such globally integrable form $\omega$.

One of the main achievement of [8] is to extend this proof to the case where $\omega$ cannot be written globally as $\phi^{*} d x_{1} \wedge d x_{2}$. In such a situation one does not have anymore a nice indexation of the leaves, defined by $\omega$, by the values of a map $\phi$, the coarea formula cannot be applied and the decomposition of the Lebesgue measure on $T$ as the measure $\frac{d \mathcal{H}^{1}}{|\omega|}$ along each leaf times some transversal measure, does not necessarily exists (in particular because a.e. leaf, for the transverse measure $\omega$, is not necessarily compact and one would have to find a measure on the space of leaves that can be already particularly complicated, see [5]). The idea used to overcome this difficulty, originally appeared in a paper by V.I. Arnold [1], where he gives an ergodic description of what he called the asymptotic Hopf invariant. He introduced the flow $g_{t}$ of the divergence free vector field $X$ associated to $\omega$ (i.e. for all vector $Y$ $<* \omega, Y>=(X, Y)$ where $<,>$ means the duality between forms and vector and $($,$) is the scalar product in \mathbb{R}^{3}$ ), this flow preserves the volume form and he replaced somehow the decomposition of the Lebesgue measure given by the coarea formula we had in the case $\omega=\phi^{*} d \sigma$, by the integration over the line flows of $X$ : 


$$
\begin{aligned}
\forall t>0 \quad \forall f \in L^{1}(T) \\
\int_{T} f=\int_{T} \frac{1}{t} d x \int_{0}^{t} f\left(g_{s}(x)\right) d s
\end{aligned}
$$

This elementary identity means that, in order to integrate a function over the space $T$, one can start to take the mean value of $f$ on the leaf starting at $x$ up to $g_{t}(x)$ for the measure $d t=\frac{d \mathcal{H}^{1}}{|X|}$ and then, integrate these time averages over all of $T$ relatively to the Lebesgue measure. Moreover, by the mean of Birkhoff Ergodic theorem, the time average in the integral of the right-hand-side of (1.7) converges almost everywhere. Precisely this idea was used in the following way. The Hopf invariant of a $C^{1}$ map $u$ from $S^{3}$ into $S^{2}$ is given by

$$
H(u)=\int_{S^{3}} u^{*} \omega \wedge \eta
$$

where $\omega$ is any closed 2-form generating $H^{2}\left(S^{2}\right)$, normalized such that $\int \omega=1$ and $\eta$ is any 1 -form such that $d \eta=u^{*} \omega$. The use of the coarea formula as above yields

$$
H(u)=\frac{1}{4 \pi^{2}} \int_{S^{2}} \int_{S^{2}} \mathrm{lk}\left(u^{-1}(\xi) ; u^{-1}(\zeta)\right) d \xi d \zeta
$$

where lk denotes the linking number between two curves of $S^{3}$. In [1] V.I. Arnold considers any closed 2-form on $S^{3}$ and the generalized the Hopf invariant also called the helicity of $d A$, introduced in hydrodynamics by H.K.Moffatt (see [15]),

$$
\mathcal{H}(d A)=\int_{S^{3}} d A \wedge A
$$

and he gave the following ergodic interpretation for $\mathcal{H}$

$$
\mathcal{H}(d A)=\int_{S^{3}} \int_{S^{3}} \lambda(x ; y) d x d y
$$

where $\lambda(x ; y)$ is the following average of linking numbers for a.e. $(x, y)$

$$
\lambda(x ; y)=\lim _{T \rightarrow+\infty} \frac{1}{T^{2}} \int_{s=0}^{T} \int_{t=0}^{T} \operatorname{lk}\left(\Gamma_{t}(x) ; \Gamma_{s}(y)\right) d t d s
$$

and $\Gamma_{t}$ denotes the union of two paths $\Gamma_{t}(x)=G_{t}(x) \cup \Delta\left(x, g_{t}(x)\right) . G_{t}(x)$ is the flow line of the divergence free vector associated to $d A$ between $x$ and $g_{t}(x)$ ( $g_{t}$ denotes the flow itself) and $\Delta$ is an assignment of a smooth curve $\Delta(x, y)$ to every couple $(x, y)$ that connects the points $x$ and $y$ such that it depends on $x$ and $y$ in a measurable way and such that the integral of the Gauss-form $\alpha$ over the product of any two such curves or over the product of any such a curve with a $G_{t}(x)(t \leq 1)$ are uniformly bounded. Recall that the Gauss-form living in $\Lambda^{2}\left(\mathbb{R}^{3} \times \mathbb{R}^{3}\right)$ is given by

$$
\alpha=\sum_{i=1}^{3} \frac{1}{4 \pi} \frac{x_{i}-y_{i}}{|x-y|^{2}} d x_{i+1} \wedge d y_{i-1}
$$

where indices $i$ are in $\mathbb{Z}_{3}$. Such assignment of curves $\Delta$ is called a "system of short paths" and it is proved that the limit $\lambda$ is independent of the choice of such a $\Delta$.

Combining this approach and the proof we gave above of (1.1) in the particular case where $\omega$-a.e leaves are compact, one can extend the lower bound to general $\omega$ (see [8]). 
1.2. High dimensional helicities. At the end of [1], V.I. Arnold ask about the existence of a similar Ergodic interpretation like (1.8) but for the following higher dimensional helicities whose "compact" version was introduced by S.P.Novikov (see [16], [17]) in it's "geometric realization" of the theory of rational homotopy of D.Sullivan .

Consider 2 closed 2-forms on $S^{4} d A$ and $d B$ that are integrable

$$
d A \wedge d A=0 \quad d B \wedge d B=0
$$

and that commute

$$
d A \wedge d B=0
$$

then we introduce

$$
\mathcal{N}(d A, d B)=\left(\int_{S^{4}} d A \wedge A \wedge B ; \int_{S^{4}} d B \wedge B \wedge A\right)
$$

The "compact" version of these helicities arises as one computes the rational homotopy class of a map $u$ from $S^{4}$ into $\mathbb{R}^{3} \backslash\left\{x_{1}, x_{2}\right\}$ where $x_{1}$ and $x_{2}$ are two separated points of $\mathbb{R}^{3}$. The infinite part of $\pi_{4}\left(\mathbb{R}^{3} \backslash\left\{x_{1}, x_{2}\right\}\right)$ is $\mathbb{Z} \oplus \mathbb{Z}$ and the corresponding class to $u$ is given by

$$
\left(\int_{S^{4}} u^{*} \omega_{1} \wedge \eta_{1} \wedge \eta_{2} ; \int_{S^{4}} u^{*} \omega_{2} \wedge \eta_{2} \wedge \eta_{1}\right)
$$

where $d \eta_{1}=u^{*} \omega_{1}, d \eta_{2}=u^{*} \omega_{2}$, and $\omega_{1}$ and $\omega_{2}$ are two 2-forms generating $H^{2}\left(\mathbb{R}^{3} \backslash\right.$ $\left.\left\{x_{1}, x_{2}\right\}\right)$ and normalized such that $\int_{\sigma_{i}} \omega_{i}=1\left(\sigma_{i}\right.$ is any sphere around $\left.x_{i}\right)$.

In part I we give an interpretation of this integers in terms of the relative linking number of preimages by $u$ of certain subparts of $\mathbb{R}^{3} \backslash\left\{x_{1}, x_{2}\right\}$ (see proposition 2.3). The relative linking number of a triplet $\left(\Sigma_{0}, \Sigma_{1}, \Sigma_{2}\right)$ of three closed disjoint surfaces of $\mathbb{R}^{4}$ is the topological degree of the following map

$$
\begin{gathered}
V: \Sigma_{0} \times \Sigma_{1} \times \Sigma_{2} \longrightarrow S^{3} \times S^{3} \\
(x, y, z) \longrightarrow\left(\frac{x-y}{|x-y|}, \frac{x-z}{|x-z|}\right) \\
\operatorname{rlk}\left(\Sigma_{0} \mid \Sigma_{1}, \Sigma_{2}\right):=\operatorname{deg}(V)
\end{gathered}
$$

See figure 1 for a non trivial relative linking number between 3 torii in $\mathbb{R}^{4}$.

Going back to the non-compact version of the Novikov helicities (1.12) we observe first that since $d A \wedge d A=0$, Darboux theorem says that, in a neighborhood of a point where $d A$ does not vanish, it may be written as $d A=\phi^{*} d x \wedge d y$ where $\phi$ is a submersion into $D^{2}$ (i.e. the kernel of $d A$ is an integrable distribution of 2-planes). Thus $d A$ defines a foliation in $S^{4}$ away from the set where $d A$ vanishes. The leaf $\mathcal{L}^{A}(x)$ of this almost foliation defined by $d A$ passing through $x \in S^{4}$, where $d A(x) \neq 0$, is the set

$$
\begin{aligned}
\mathcal{L}^{A}(x)=\left\{y \in S^{4} \quad \text { s.t. } \exists \gamma \in C^{1}\left([0,1] ; \mathcal{T}_{i}\right)\right. & \gamma(0)=x \gamma(1)=y \\
& \left.\forall t \in[0,1] \quad d A_{\gamma(t)} \neq 0 \quad ; \quad \dot{\gamma}(t) \in \operatorname{Ker}\left(d A_{\gamma(t)}\right)\right\}
\end{aligned}
$$



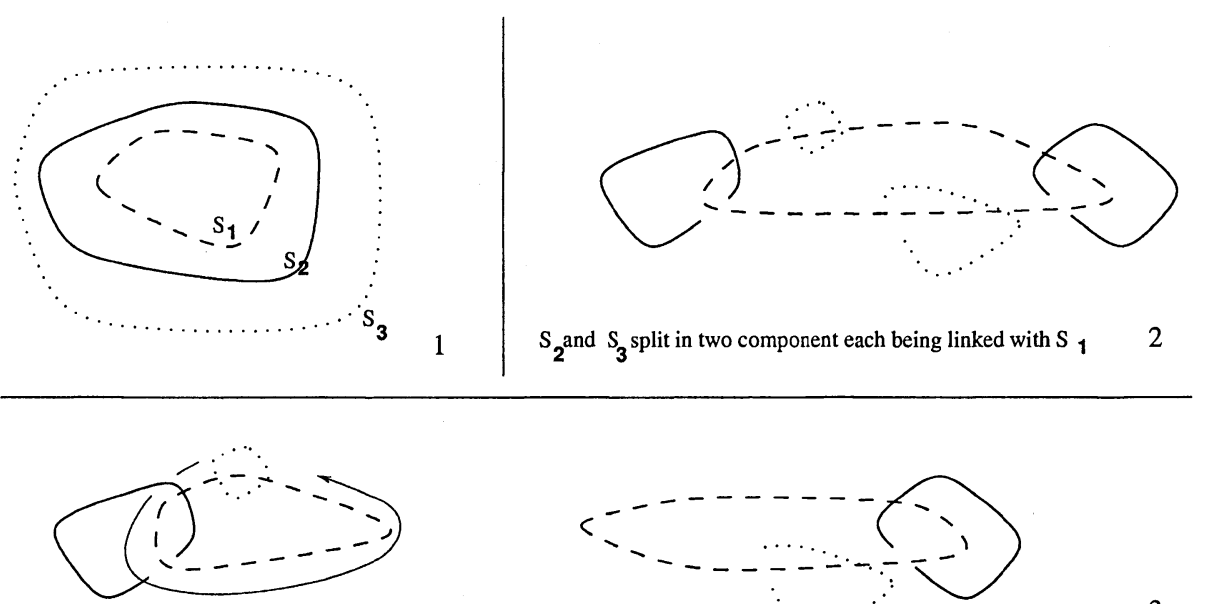

$S_{Y}$ splits and one of the component of $S_{3}$ the left one here) makes one lap

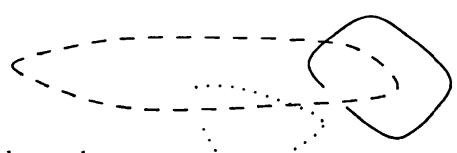

around the component of $S_{j}$ it is linked with, passing inside the component of $S$ and comes back to its original position.

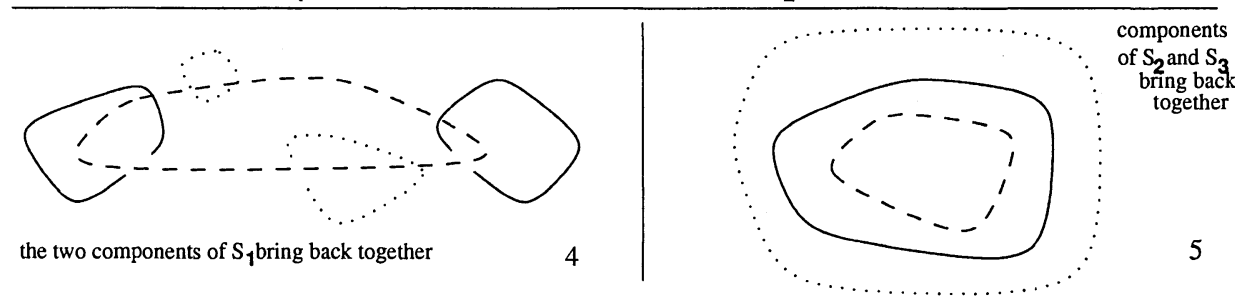

FIG. 1.1. relative linking numbers of 3 torii $S_{1}, S_{2}$ and $S_{3}$ in $\mathbb{R}^{4}$ : picture 1 to 5 represents 5 slicing increasing in time of $\mathbb{R}^{4}$, in that case we have $\operatorname{rlk}\left(S_{3} \mid S_{2}, S_{1}\right)=1$ providing a right orientation of each torus - observe that $\operatorname{rlk}\left(S_{2} \mid S_{3}, S_{1}\right)=0$.

Let $\mathcal{X}$ be the set of oriented 2-submanifolds of $S^{4}$ which are everywhere transverse to the leaves, then $d A$ defines a positive invariant measure over $\mathcal{X}$ (see [18]). We say that some property holds for $d A$-a.e. leaves of $d A$ if for any $N$ in $\mathcal{X}$ the measure relative to $d A$ of the $x$ in $N$ such that the property does not hold for $\mathcal{L}^{A}(x)$ is 0 .

DEFInITION 1.1. The zero set of $d A$ is said to be $d A$-negligible if for $d A$ almost every leaf the zero set of $d A$ has no intersection with the closure of the leaf. The foliation defined by $d A$ is then called a lamination.

Definition 1.2. A point $x$ in $S^{4}$ is said to be essential for the lamination defined by $d A$ if the leaf through $x$ does not intersect the closure of $\operatorname{KerdA}$.

We will assume from now on that both $d A$ and $d B$ define laminations.

We will need some "closing at infinity" for $d A$-a.e. leaf of $d A$. A leaf $\mathcal{L}^{A}(x)$ is said to be of Liouville type if there exists no non-constant harmonic function on $\mathcal{L}^{A}(x)$. For instance, a sufficient condition for beeing Liouville is, for the leaf $\mathcal{L}^{A}(x)$, to have subexponential growth (see [12]) i.e.

$$
\forall y \in \mathcal{L}^{A}(x) \quad \lim _{R \rightarrow+\infty} \frac{\log \left|B_{R}(y)\right|}{R} \longrightarrow 0
$$

where $B_{R}(y)$ denotes the geodesic ball in $\mathcal{L}^{A}(x)$ of center $y$ and radius $R$. The characterization that we will use of being of Liouville type for $\mathcal{L}$ is the cancellation of 
the Kaimanovich Entropy on $\mathcal{L}$ that was proved in [11] and [12] :

$$
\lim _{t \rightarrow \infty} \frac{1}{t} \int_{\mathcal{L}} p(x, y, t) \log p(x, y, t) d \mathcal{H}^{2}(y)=0
$$

for any $x$ on $\mathcal{L}$ and where $p$ is the Heat-Kernel for the laplacian on $\mathcal{L}$.

Definition 1.3. An integrable 2-form $d A$ defines a Liouville lamination if it defines a lamination and if $d A$-a.e. leaf of this lamination is of Liouville type.

REMARK 1.1. Liouville laminations which are not necessarily compact are easy to produce. One takes a non degenerate closed two forms in a 2-torus contained $\mathbb{R}^{3}$ minus the Ox-axis. Assume this two form is tangent to the boundary of the torus and defines a non compact foliation inside. Then the non-compact Liouville lamination is obtained by rotating this foliation in $\mathbb{R}^{4}$ about the $O x$ axis.

Consider now two 2-forms $d A$ and $d B$ verifying (1.10) and (1.11) each of these two defining a Liouville Lamination ( (1.11) implies in particular that $d A \times d B$-a.e. pairs of $d A$ - and $d B$-leaves do not intersect - see lemma 2.1). Let $x$ be an essential point for $d A$ we denote by $p_{A}$ the heat-kernel on the leaf passing through $x, \mathcal{L}^{A}(x)$, for the metric induced on this leaf from $g_{S^{4}} /|d A|$. Let $\delta$ be any positive number, we define

$$
\mathcal{L}_{k, \delta}^{A}(x, t)=\mathcal{L}^{A}(x) \cap\left\{\xi ; \delta^{k+1} \leq p_{A}(x, \xi, t) \leq \delta^{k}\right\}
$$

and

$$
\mathcal{D}_{k, \delta_{T}}^{A}(x, t)=\mathcal{L}_{k, \delta_{T}}^{A}(x, t) \cup C_{\partial \mathcal{L}_{k, \delta_{T}}^{A}(x, t)}
$$

where $C$ denotes an assignment of surfaces to any closed curve lying in a leaf of $d A$ called a "system of small caps" (see proposition-definition 2.1) replacing somehow the "system of short paths" $\Delta$ of the 1-D case. Let $\delta_{T}$ be a positive function tending to 1 as $T \rightarrow+\infty$. We define $\Lambda^{T}$ to be the following relative linking average

For $(x, y, z)$ being an essential triplet for rep. $d A, d A$ and $d B$

$$
\begin{aligned}
& \forall(k, l, m) \in \mathbb{N}^{3} \text { let } \\
& \Lambda_{k, l, m}^{T}(x, y, z)=\frac{1}{T} \int_{[0, T]^{3}} \operatorname{rlk}\left(\mathcal{D}_{k, \delta_{T}}^{A}(x, t) \mid \mathcal{D}_{l, \delta_{T}}^{A}(y, s) ; \mathcal{D}_{m, \delta_{T}}^{B}(z, \sigma)\right) d t d s d \sigma
\end{aligned}
$$

and define

$$
\begin{aligned}
& \Lambda^{T}(x, y, z)=\sum_{k, l, m} \delta_{T}^{k+l+m} \Lambda_{k, l, m}^{T}(x, y, z) \\
& \Lambda^{T}(x, y, z)=0 \quad \text { otherwise }
\end{aligned}
$$

We can state now our first main result

ThEOREM 1.1. Assume $d A$ and $d B$ are two integrable 2-forms of $S^{4}$ that commute, $d A \wedge d B=0$, assume they both define Liouville laminations then the following ergodic interpretation of the Novikov helicity holds : there exists $\delta_{T} \rightarrow 1$ such that

$$
\lim _{T \rightarrow+\infty} \Lambda^{T}(x, y, z)=\Lambda(x, y, z) \quad \text { exists for a.e. }(x, y, z)
$$


The limit is independent on the "system of small caps" chosen, moreover we have

$$
\int_{S^{4}} d A \wedge A \wedge B=\int_{S^{4}} \int_{S^{4}} \int_{S^{4}} \Lambda(x, y, z)
$$

An ergodic interpretation of the Hopf-Novikov helicities $\int_{S^{4}} d A \wedge A \wedge B$ was already obtained by B.A. Khesin in [13] and involves the notion of assymptotic linking between divergence free fields and foliations (see more about this approach in [14]). This ergodic interpretation is based on the following observation : since $d A \wedge d B=0$ generically as two leaves $\mathcal{L}^{A}(x)$ and $\mathcal{L}^{B}$ intersect the resulting intersection is a line. The 1-dim "almost foliation" obtained is given by the flow of the divergence-free field associated to the form $d A \wedge B$. Then $\int d A \wedge B \wedge A$ is interpreted as a mean value of averages of linking numbers between the 1-dim lines of the foliation $d A \wedge B$ and the foliation given by $d A$. Again Arnolds approach via the flow along the divergence-free field associated to $d A \wedge B$ and the system of short path $\Delta$ is used but no ergodic interpretation of the linking number between a curve and the 2-dimensional "almost foliation" generated by $d A$ is given. Using our approach, such an interpretation can be given in terms of averages of linking between curves and surfaces in the case where $d A$ defines a Liouville lamination.

In particular our approach works also for the simplest helicity $\int d A \wedge A$, the Liouville restriction is not needed in this case since the leaves are 1-dimensional. The lamination condition was also required for the existence of short paths (see remark 4.14 page 145 of [2]) until the recent paper by T. Vogel [19].

Still for $M=\mathbb{R}^{3} \backslash\left\{x_{1}, x_{2}\right\}$ one can iterate the procedure of computing the continuation algebras $C_{q}(A)$ of the minimal model $A$ of $\wedge^{*} M$ (see [16] and [17]) in order to obtain all the geometric realizations of the rational homotopy class of $\mathbb{R}^{3} \backslash\left\{x_{1}, x_{2}\right\}$. For the $\pi_{4}\left(\mathbb{R}^{3} \backslash\left\{x_{1}, x_{2}\right\}\right) \otimes \mathbb{Q} \simeq \mathbb{Q}^{2}$ these geometric realizations are $u^{*} \omega_{1} \wedge \eta_{1} \wedge \eta_{2}$ and $u^{*} \omega_{2} \wedge \eta_{1} \wedge \eta_{2}$ that we saw before. One step further, for a map $u: S^{5} \longrightarrow \mathbb{R}^{3} \backslash\left\{x_{1}, x_{2}\right\}$, the geometric realization, induced by $u$, of the generators of the $H^{5}\left(C_{5}(A)\right)$ gives the following 3 homotopic invariants corresponding to $\pi_{5}\left(\mathbb{R}^{3} \backslash\left\{x_{1}, x_{2}\right\}\right) \otimes \mathbb{Q} \simeq \mathbb{Q}^{3}$ : $u^{*} \omega_{1} \wedge \eta_{1} \wedge d^{-1}\left(u^{*} \omega_{1} \wedge \eta_{2}\right), u^{*} \omega_{2} \wedge \eta_{2} \wedge d^{-1}\left(u^{*} \omega_{1} \wedge \eta_{2}\right)$ and $u^{*} \omega_{1} \wedge \eta_{2} \wedge d^{-1}\left(u^{*} \omega_{1} \wedge \eta_{2}\right)$. The method we develop permits to give a geometric interpretation of these invariants in terms of higher order linkings with a corresponding Gauss integral formulas...etc. Moreover our method yields an ergodic interpretation of the following helicities in $S^{5}$ :

$$
\int_{S^{5}} d B \wedge B \wedge C \quad \int_{S^{5}} d A \wedge A \wedge C \quad \int_{S^{5}} d A \wedge B \wedge C
$$

where we assume that $A \in \wedge^{1} S^{5}, B \in \wedge^{1} S^{5}$ verify

$$
d A \wedge d A=0 \quad d B \wedge d B=0 \quad d A \wedge d B=0 \quad \text { and } d C=d A \wedge B
$$

These helicities are again just examples and we claim that the approach we develop for the helicity above (1.12) can be transposed to all the helicities one can construct from the Hopf-Novikov Invariants that give integral representation of the rational homotopy groups described in Sullivan Theory of the minimal models.

Passing from 1 dimensional leaves to 2 or any higher dimensional leaves required the introduction of a new way of "moving around over the leaves" compare to the one given by the flow of the divergence free field associated to the form in 3 dimension. This way of moving has to "decompose" the Lebesgue measure in the ambient space (like the coarea in the case of compact leaves) into a measure along the leaf times a 
measure over the set parameterizing the leaves. In the 1 dimensional case (1.7) the set of leaves was trivially over-parametrised by the all set of points of the ambient space. One of our main observation will be to interpret divergence free motion and the corresponding Birkhoff Ergodic Theorem as a motion on the leaf which is deterministic among motions of more general nature with more general ergodic theorem : we have

$$
\int_{S^{4}} f=\int_{S^{4}} \frac{1}{t} \int_{0}^{t} d \tau \int_{\text {Leaf }} f(y) p(x, y, \tau)
$$

where $p(x, y, \tau)=\delta_{\Phi_{\tau}(x)}$. Our idea is to replace this deterministic motion, which has no meaning for 2-dimensional leaves, by some Brownian motion on the leaves which is not deterministic anymore : we will look for a probability measure $p(x, d y, t)$, see theorem 2.1 , verifying the three following conditions

i) $p(x, d y, t)$ is supported on the leaf passing through $x$.

ii) If the leaf passing through $x, \mathcal{L}(x)$ is compact, for every function $f$

$$
\lim _{t \rightarrow+\infty} \int_{\mathcal{L}(x)} f(y) p(x, d y, t) \longrightarrow c_{\mathcal{L}(x)} \int_{\mathcal{L}(x)} f(y) \frac{d \mathcal{H}^{2}}{|d A|}
$$

iii) (preservation of the Lebesgue measure) For every function $f$

$$
\int_{S^{4}} d x \frac{1}{t} \int_{0}^{t} d \tau \int_{S^{4}} f(y) p(x, d y, \tau) \longrightarrow \int_{S^{4}} f(x) d x
$$

where $d x$ denotes the Lebesgue measure. Moreover we will require to have an Ergodic property saying that the average in time in the left-hand side of the previous equality converges almost everywhere. We will view the introduction of such a decomposition of the Lebesgue measure as a substitute of the coarea formula in the case where the leaves are not necessarily compact and not necessarily indexed as preimages of points by some map.

1.3. Rigidity of linked Liouville Laminations. In this part we will apply the technics developed to solve theorem 1.1 and to give the Ergodic interpretation of Hopf-Novikov's Invariants to get topological lower-bounds for conformal invariant energies of differential forms (such as the $L^{2}$ scalar product of 2-forms in 4 dimensions) defining Liouville Laminations in the spirit of estimate (1.1). To this aim we need to introduce few topological invariants.

Let $\Sigma_{1}, \Sigma_{2}$ and $\Sigma_{3}$ three closed disjoint (not necessarily connected) surfaces in $\mathbb{R}^{4}$ (or $S^{4}$ ), we define the relative over-crossing number, $\operatorname{rc}\left(\Sigma_{1} \mid \Sigma_{2}, \Sigma_{3}\right)$, of $\Sigma_{2}$ and $\Sigma_{3}$ relative to $\Sigma_{1}$ in the following way. For almost every vector $u$ in $S^{3}$ the projection of $\Sigma_{2}$ and $\Sigma_{1}$ on a plane perpendicular to $u$ are transverse to each other and for such a $u$ we define the "shadow" of $\Sigma_{2}$ on $\Sigma_{1}$ to be the following set

$$
S_{u}\left(\Sigma_{1}, \Sigma_{2}\right)=\left\{x \in \Sigma_{1} \quad \text { s.t. } \quad \exists y \in \Sigma_{2} \quad u=\frac{x-y}{|x-y|}\right\}
$$

Take now another "generic" vector $v$ of $S^{3}$ such that the projection of $S_{u}$ and $\Sigma_{3}$ on a plane perpendicular to $v$ are transverse to each other. The over-crossing set of $S_{u}$ and $\Sigma_{3}$ relative to $v$ is

$$
S_{u, v}\left(\Sigma_{1} \mid \Sigma_{2}, \Sigma_{3}\right)=\left\{x \in S_{u} \quad \text { s.t. } \quad \exists z \in \Sigma_{3} \quad v=\frac{x-z}{|x-z|}\right\}
$$


The over-crossing number of $\Sigma_{2}$ and $\Sigma_{3}$ relative to $\Sigma_{1}$ is the minimum among all smooth deformations of $\mathbb{R}^{4}$ and among almost every "generic" $u$ and $v$ of the cardinal of $S_{u, v}$

$$
\operatorname{rc}\left(\Sigma_{1} \mid \Sigma_{2}, \Sigma_{3}\right)=\min _{\text {a. e. } u, v \in S^{3} ; \psi \in \text { diffeo. } \mathbb{R}^{4}} \operatorname{Card}\left(S_{u, v}\left(\psi\left(\Sigma_{1}\right) \mid \psi\left(\Sigma_{2}\right), \psi\left(\Sigma_{3}\right)\right)\right)
$$

In the same spirit of [8] we can define the asymptotic relative crossing number of $\Sigma_{2}$ and $\Sigma_{3}$ relative to $\Sigma_{1}, \operatorname{arc}\left(\Sigma_{1} \mid \Sigma_{2}, \Sigma_{3}\right)$ to be the following number . We assume in a first approach that each of the 3 surfaces $\Sigma_{1}, \Sigma_{2}$ and $\Sigma_{3}$ are connected. Let $\mathcal{T}_{1}$, $\mathcal{T}_{2}$ and $\mathcal{T}_{3}$ be 3 disjoint tubular neighborhoods of $\Sigma_{1}, \Sigma_{2}$ and $\Sigma_{3}\left(\mathcal{T}_{i} \simeq \Sigma_{i} \times D^{2}\right)$ in $\mathbb{R}^{4}$. Inside $\mathcal{T}_{1}, \mathcal{T}_{2}$ and $\mathcal{T}_{3}$ respectively take $\Sigma_{1}^{\prime}, \Sigma_{2}^{\prime}$ and $\Sigma_{3}^{\prime}$ to be 3 closed surfaces (not necessarily connected) such that

$$
\operatorname{deg}\left(\Sigma_{i}^{\prime} ; \mathcal{T}_{i}\right):=\left[\Sigma_{i}^{\prime}\right] \cdot D_{i} \neq 0
$$

where $\left[\Sigma_{i}^{\prime}\right.$ ] is the homology class in $H_{2}\left(\mathcal{T}_{i}\right)$ of $\Sigma_{i}^{\prime}, D_{i}$ is the homology class in $H_{2}\left(\mathcal{T}_{i}, \partial\right)$ obtained from any section of $\mathcal{T}_{i}$ whose intersection number with $\left[\Sigma_{i}\right]$ is +1 ( $\cdot$ is the intersection numbers operation). Then we define

$$
\begin{aligned}
& \operatorname{arc}\left(\Sigma_{1} \mid \Sigma_{2}, \Sigma_{3}\right)= \\
& \min \left\{\begin{array}{lcc}
\operatorname{rc}\left(\Sigma_{1}^{\prime} \mid \Sigma_{2}^{\prime}, \Sigma_{3}^{\prime}\right) & \text { s. t. } & \mathcal{T}_{i} \text { disjoint tubu. neigh. of } \Sigma_{i}^{\prime} \\
\prod_{i=1}^{3} \mid \operatorname{deg}\left(\Sigma_{i}^{\prime} ; \mathcal{T}_{i}\right) & \Sigma_{i}^{\prime} \text { clos. surfaces in } \mathcal{T}_{i}
\end{array}\right\}
\end{aligned}
$$

where the minimum is also taken among all smooth deformations of $\mathbb{R}^{4}$.

In the case where $\Sigma_{1}, \Sigma_{2}$ and $\Sigma_{3}$ are not connected anymore the definitions have to be changed. For instance, if $\Sigma_{1}$ and $\Sigma_{2}$ have 1 connected component each but $\Sigma_{3}$ has 2 connected components $\tilde{S}_{3}$ and $\hat{S}_{3}, \operatorname{arc}\left(\Sigma_{1} \mid \Sigma_{2}, \Sigma_{3}\right)$ is defined in the following way : for any choice of four surfaces $\Sigma_{1}^{\prime}, \Sigma_{2}^{\prime}, \tilde{\Sigma}_{3}^{\prime}$ and $\hat{\Sigma}_{3}^{\prime}$ in $\mathcal{T}_{1}, \mathcal{T}_{2}, \tilde{T}_{3}$ and $\hat{T}_{3}$ we consider the minimum among any generic $u$ and $v$ of the sum

$$
\frac{S_{u, v}\left(\Sigma_{1}^{\prime} \mid \Sigma_{2}^{\prime}, \tilde{\Sigma}_{3}^{\prime}\right)}{\left|\operatorname{deg}\left(\Sigma_{1}^{\prime}, \mathcal{T}_{1}\right) \operatorname{deg}\left(\Sigma_{2}^{\prime}, \mathcal{T}_{2}\right) \operatorname{deg}\left(\tilde{\Sigma}_{3}^{\prime}, \mathcal{T}_{3}\right)\right|}+\frac{S_{u, v}\left(\Sigma_{1}^{\prime} \mid \Sigma_{2}^{\prime}, \hat{\Sigma}_{3}^{\prime}\right)}{\left|\operatorname{deg}\left(\Sigma_{1}^{\prime}, \mathcal{T}_{1}\right) \operatorname{deg}\left(\Sigma_{2}^{\prime}, \mathcal{T}_{2}\right) \operatorname{deg}\left(\hat{\Sigma}_{3}^{\prime}, \mathcal{T}_{3}\right)\right|}
$$

and the minimum of such quantity among all possible choices of $\Sigma_{1}^{\prime}, \Sigma_{2}^{\prime}, \tilde{\Sigma}_{3}^{\prime}$ and $\hat{\Sigma}_{3}^{\prime}$ in $\mathcal{T}_{1}, \mathcal{T}_{2}, \tilde{T}_{3}$ and $\hat{T}_{3}$ and all possible smooth deformations gives $\operatorname{arc}\left(\Sigma_{1} \mid \Sigma_{2}, \Sigma_{3}\right)$.

Our second main result reads.

Theorem 1.2. Let $\mathcal{T}_{1}, \mathcal{T}_{2}$ and $\mathcal{T}_{3}$ be three disjoint tubular neighborhoods of three disjoint closed surfaces $\Sigma_{1}, \Sigma_{2}$ and $\Sigma_{3}$ in $\mathbb{R}^{4}$. Let $d A_{i}$ be three integrable 2-forms $\left(d A_{i} \wedge d A_{i}=0\right)$ in $\mathcal{T}_{i}$ defining Liouville laminations and so that $\iota_{\partial \mathcal{T}_{i}}^{*} d A_{i}=0\left(\iota_{\partial \mathcal{T}_{i}}\right.$ is the inclusion map of $\partial \mathcal{T}_{i}$ in $\mathbb{R}^{4}$ ) then the following identity holds

$$
\begin{gathered}
\frac{1}{16 \pi^{4}} \int_{\mathcal{T}_{1}} \int_{\mathcal{T}_{2}} \int_{\mathcal{T}_{3}} \frac{\left|d A_{1}\right|(x)\left|d A_{2}\right|(y)\left|d A_{3}\right|(z)}{|x-y|^{3}|x-z|^{3}} \geq \\
\prod_{i=1}^{3}\left|\operatorname{Flux}\left(d A_{i}\right)\right| \operatorname{arc}\left(\Sigma_{1} \mid \Sigma_{2}, \Sigma_{3}\right)
\end{gathered}
$$

where Flux $\left(d A_{i}\right)$ is the integral over any oriented transverse section of $\mathcal{T}_{i}$ having intersection number +1 with $\Sigma_{i}$. 
It is clear that

$$
\operatorname{arc}\left(\Sigma_{1} \mid \Sigma_{2}, \Sigma_{3}\right) \geq\left|\operatorname{rlk}\left(\Sigma_{1} \mid \Sigma_{2}, \Sigma_{3}\right)\right|
$$

Inequality (1.21) with $\operatorname{rlk}\left(\Sigma_{1} \mid \Sigma_{2}, \Sigma_{3}\right)$ instead of $\operatorname{arc}\left(\Sigma_{1} \mid \Sigma_{2}, \Sigma_{3}\right)$ does not require the $d A_{i}$ to be integrable and follows by standard cohomological arguments using Poincaré duality (see for instance [3]) and the integral formula we give bellow for the relative linking number. In the last section we give an example of surfaces where

$$
\operatorname{rlk}\left(\Sigma_{1} \mid \Sigma_{2}, \Sigma_{3}\right)=0 \quad \text { and } \quad \operatorname{arc}\left(\Sigma_{1} \mid \Sigma_{2}, \Sigma_{3}\right)>0
$$

The asymptotic crossing number may be compared with other topological invariants : Consider any smooth 3-manifold $M_{2}$ bounding $\Sigma_{2}\left(\partial M_{2}=\Sigma_{2}\right)$ and intersecting $\Sigma_{1}$ transversally. The intersection $M_{2} \cap \Sigma_{1}$ defines an homology class $\sigma_{1,2}$ in $H_{1}\left(\mathcal{T}_{1}\right)$ which is independent on $M_{2}$ verifying $\partial M_{2}=\Sigma_{2}$. This is nothing but the intersection pairing between the class defined by such an $M_{2}$ in $H_{3}\left(\mathbb{R}^{4}, \Sigma_{2}\right)$ and $H_{2}\left(\Sigma_{1}\right)$ (see [6] page 336). Taking now the restriction to $\mathbb{R}^{4} \backslash \mathcal{T}_{3}$ of an immersed surface that bounds a smooth representant of $\sigma_{1,2}$, it defines a class $\sigma_{1,2,3}$ in $H_{2}\left(\mathbb{R}^{4} \backslash \mathcal{T}_{3}, \Sigma_{1} \cup \mathcal{T}_{3}\right)$ which is independent of the representant of $\sigma_{1}$ chosen (since $\Sigma_{1} \cap \mathcal{T}_{3}=\emptyset$ ). Observe that the boundary part of $\sigma_{1,2,3}$ in $H_{1}\left(\partial \mathcal{T}_{3}\right)$ intersected with $M_{3}$, a manifold bounding $\Sigma_{3}$, gives the relative linking number $\operatorname{rlk}\left(\Sigma_{1} \mid \Sigma_{2}, \Sigma_{3}\right)$. Then we prove

THEOREM 1.3. Let $\sigma_{1,2,3}$ being the class in $H_{2}\left(\mathbb{R}^{4} \backslash \mathcal{T}_{3} ; \Sigma_{1} \cup \partial \mathcal{T}_{3}\right)$ defined above, we have

$$
\operatorname{arc}\left(\Sigma_{1} \mid \Sigma_{2}, \Sigma_{3}\right) \geq\left\|\sigma_{1,2,3}\right\|
$$

where \|\| denotes the singular pseudo-norm in $H_{2}\left(\mathbb{R}^{4} \backslash \mathcal{T}_{3} ; \Sigma_{1} \cup \partial \mathcal{T}_{3}\right)$

$$
\begin{aligned}
\left\|\sigma_{1,2,3}\right\|=\inf \{1 / n x(S) \mid f: S \rightarrow M \quad \text { and } \\
\left.f_{*}[S]=n \sigma_{1,2,3} \text { in } H_{2}\left(\mathbb{R}^{4} \backslash \mathcal{T}_{3} ; \Sigma_{1} \cup \partial \mathcal{T}_{3}\right)\right\} .
\end{aligned}
$$

and $x(S)=\sum_{i} \chi_{-}\left(S_{i}\right)$ where $S_{i}$ are the connected components of $S, \chi_{-}\left(S_{i}\right)=$ $\max \left\{0,-\chi\left(S_{i}\right)\right\}$ and $\chi$ is the Euler characteristic.

\section{Preliminaries.}

2.1. The 4-dimensional Gauss formula for relative linkings. Let $\Sigma_{0}, \Sigma_{1}$ and $\Sigma_{2}$ be three closed surfaces in $\mathbb{R}^{4}$ such that $\Sigma_{0} \cap \Sigma_{1}=\emptyset$ and $\Sigma_{0} \cap \Sigma_{2}=\emptyset$. $\operatorname{rlk}\left(\Sigma_{0} \mid \Sigma_{1}, \Sigma_{2}\right)$ is the relative linking number of this triplet defined by (1.15). The following Gauss formula for the relative linking holds.

Proposition 2.1. We have

$$
\operatorname{rlk}\left(\Sigma_{0} \mid \Sigma_{1}, \Sigma_{2}\right)=\int_{\Sigma_{0} \times \Sigma_{1} \times \Sigma_{2}} \mathcal{G}
$$

where $\mathcal{G}$ is the following Gauss-form defined on $\left(\mathbb{R}^{4}\right)^{3}$

$$
\mathcal{G}=\frac{1}{4 \pi^{4}} \sum_{i \neq k} \sum_{j \neq l} \frac{x_{i}-y_{i}}{|x-y|^{4}} \frac{x_{j}-z_{j}}{|x-z|^{4}} d x_{k} d x_{l} *\left(d y_{i} d y_{k}\right) *\left(d z_{j} d z_{l}\right)
$$


Proof of proposition 2.1. Let $\Omega=\frac{1}{\left|S^{3}\right|} \sum_{i=1}^{4} X_{i}\left(d X_{1} . . d X_{4}\right)_{i}$ where we will use the notation $\left(d X_{1} . . d X_{4}\right)_{i}:=\iota_{e_{i}}\left(d X_{1} d X_{2} d X_{3} d X_{4}\right)$ and $\iota_{e_{i}}$ is the interior product with the $\mathrm{i}$-th vector $e_{i}$ of the canonical basis of $\mathbb{R}^{4}$. $\Omega$ is the volume form on $S^{3}$ normalized in such a way that $\int_{S^{3}} \omega=1$. The integral representation of the topological degree of $V$ defined by (1.14) is

$$
\begin{aligned}
& \operatorname{deg}(V)=\int_{\Sigma_{0} \times \Sigma_{1} \times \Sigma_{2}} V^{*} \Omega(X) \wedge \Omega(Y) \\
& =\frac{1}{4 \pi^{4}} \int_{\Sigma_{0} \times \Sigma_{1} \times \Sigma_{2}} \sum_{i, j=1}^{4} \frac{x_{i}-y_{i}}{|x-y|^{4}} \frac{x_{j}-z_{j}}{|x-z|^{4}}\left(d\left(x_{1}-y_{1}\right) . . d\left(x_{4}-y_{4}\right)\right)_{i} \\
& \quad\left(d\left(x_{1}-z_{1}\right) . . d\left(x_{4}-z_{4}\right)\right)_{j}
\end{aligned}
$$

Few terms vanish while developing the product forms and the above integral becomes

$$
\frac{1}{4 \pi^{4}} \int_{\Sigma_{0} \times \Sigma_{1} \times \Sigma_{2}} \sum_{i \neq k} \sum_{j \neq l} \frac{x_{i}-y_{i}}{|x-y|^{4}} \frac{x_{j}-z_{j}}{|x-z|^{4}} d x_{k} d x_{l}\left(d y_{1} . . d y_{4}\right)_{i k}\left(d z_{1} . . d z_{4}\right)_{j l}
$$

which gives the result.

The following interpretation of the relative linking number illustrates perhaps better the link between $r l k$ and $a r c$.

Proposition 2.2. Let $\Sigma_{0}, \Sigma_{1}$ and $\Sigma_{2}$ be three closed surfaces in $\mathbb{R}^{4}$ such that $\Sigma_{0} \cap \Sigma_{1}=\emptyset$ and $\Sigma_{0} \cap \Sigma_{2}=\emptyset$. Let $M_{1}$ and $M_{2}$ be two 3 submanifolds of $\mathbb{R}^{4}$ such that $\partial M_{i}=S_{i}$ (for $i=1,2$ ). Assuming $M_{2}$ and $\Sigma_{0}$ intersect each other transversally along a curve $\Gamma_{2}$ and that this curve intersects $M_{1}$ transversally then

$$
\operatorname{rlk}\left(\Sigma_{0} \mid \Sigma_{1}, \Sigma_{2}\right)=\Gamma_{2} \cdot M_{1}
$$

where $\Gamma_{2} \cdot M_{1}$ is the intersection number between $\Gamma_{2}$ and $M_{1}$.

REMARK 2.1. It is not difficult to see that any Novikov integral expression of the rational homotopy class of a map from a sphere into a manifold (extending 1.13 for $\pi_{4}\left(\mathbb{R}^{3} \backslash\{p, q\}\right) \otimes \mathbb{Q}$ to arbitrary $\pi_{k}(N) \otimes \mathbb{Q}$ ) admits such an interpretation via intersection numbers between preimages of points and manifolds whose boundary are preimages of points. Such an interpretation makes a "geometric" illustration via intersection of submanifolds of the minimal model construction of Sullivan.

Proof of proposition 2.2. Let $T_{0}, T_{1}$ and $T_{2}$ be 3 disjoint tubular neighborhoods of the 3 disjoint surfaces $\Sigma_{0}, \Sigma_{1}, \Sigma_{2}$. Choose $\phi_{i}$ a smooth map from $T_{i}$ into $D^{2}$ (the 2unit disk of $\mathbb{R}^{2}$ centered at 0 ) such that $\phi\left(\partial T_{i}\right) \subset \partial D^{2}$ and the degree of $\phi_{i}$ on a given section $S_{i}$ of $T_{i}$ generating $H_{2}\left(T_{i}, \partial T_{i}\right)$ with intersection number +1 with $\Sigma_{i}$ is +1 . We have then $\iota_{\partial T_{i}}^{*} \phi_{i}^{*} \omega=0$, where $\iota_{\partial T_{i}}$ is the canonical embedding of $\partial T_{i}$ in $T_{i}$, and $\omega$ is the volume form on $D^{2}$ normalised so that $\int_{D^{2}} \omega=+1$. We have moreover $\int_{S_{i}} \phi_{i}^{*} \omega=$ 1. From the homotopy invariance of degrees we get that for any arbitrary choice $\left(p_{0}, p_{1}, p_{2}\right)$ of regular point of $\left(\phi_{0}, \phi_{1}, \phi_{2}\right)$ in $\left(D^{2}\right)^{3} r l k\left(\phi_{0}^{-1}\left(p_{0}\right) \mid \phi_{1}^{-1}\left(p_{1}\right), \phi_{2}^{-1}\left(p_{2}\right)\right)$ is independant of $\left(p_{0}, p_{1}, p_{2}\right)$ and equals $r l k\left(\Sigma_{0} \mid \Sigma_{1}, \Sigma_{2}\right)$. We then obtain by the mean 
of the Gauss formula above and the coarea formula that

$$
\begin{aligned}
& r l k\left(\Sigma_{0} \mid \Sigma_{1}, \Sigma_{2}\right)=\int_{D^{2}} \int_{D^{2}} \int_{D^{2}} r l k\left(\phi_{0}^{-1}\left(p_{0}\right) \mid \phi_{1}^{-1}\left(p_{1}\right), \phi_{2}^{-1}\left(p_{2}\right)\right) d p_{0} d p_{1} d p_{2} \\
& =\int_{D^{2}} \int_{D^{2}} \int_{D^{2}} d p_{0} d p_{1} d p_{2} \int_{\phi_{0}^{-1}\left(p_{0}\right) \times \phi_{1}^{-1}\left(p_{1}\right) \times \phi_{2}^{-1}\left(p_{2}\right)} \mathcal{G} \\
& =\int_{T_{0} \times T_{1} \times T_{2}} \phi_{0}^{*} \omega(x) \wedge \phi_{1}^{*} \omega(x) \wedge \phi_{2}^{*} \omega(x) \wedge \mathcal{G}
\end{aligned}
$$

Introducing $\eta_{1}$ and $\eta_{2}$ to be the following 1-forms

$$
\eta_{i}:=d^{*} \Delta^{-1} \phi_{i}^{-1}\left(p_{1}\right)=d^{*} \int_{x_{i} \in \mathbb{R}^{4}} \frac{1}{4 \pi^{2}\left|x-x_{i}\right|^{2}} \phi_{i}^{*} \omega\left(x_{i}\right)
$$

A simple computation yields that

$$
\int_{T_{0} \times T_{1} \times T_{2}} \phi_{0}^{*} \omega(x) \wedge \phi_{1}^{*} \omega\left(x_{1}\right) \wedge \phi_{2}^{*} \omega\left(x_{2}\right) \wedge \mathcal{G}=\int_{\mathbb{R}^{4}} \phi_{0}^{*} \omega \wedge \eta_{1} \wedge \eta_{2}
$$

It is a well known reslt that the $\eta_{i}$ are the Poincaré duals in $H^{1}\left(\mathbb{R}^{4} \backslash T_{i}\right)$ of any cyle bounding $\Sigma_{i}$ and the proposition follows.

2.2. Geometric interpretation of the Hopf-Novikov Invariants. We give now the interpretation of the Hopf-Novikov Invariant in terms of relative linking.

Proposition 2.3. Let $u$ be a regular map from $S^{4}$ into $\mathbb{R}^{3} \backslash\left\{p_{1}, p_{2}\right\}$ where $p_{1}$ and $p_{2}$ are two distinct points of $\mathbb{R}^{3}$. Let $\pi_{i}(i=1,2)$ be the orthogonal projection of $\mathbb{R}^{3}$ onto $S_{i}^{2}$ the unit-sphere of center $p_{i}$. Then for every regular pair of distinct points $x, y$ of $u_{1}=\pi_{1} \circ u$ and every regular point $z$ of $u_{2}=\pi_{2} \circ u$ verifying $\pi_{1}^{-1}(x) \cap \pi_{2}^{-1}(z)=\emptyset$ and $\pi_{1}^{-1}(y) \cap \pi_{2}^{-1}(z)=\emptyset$ in $\mathbb{R}^{3} \backslash\left\{p_{1}, p_{2}\right\}$, we have

$$
\int_{S^{4}} u^{*} \omega_{1} \wedge \eta_{1} \wedge \eta_{2}=r l k\left(u_{1}^{-1}(x) \mid u_{1}^{-1}(y) ; u_{2}^{-1}(z)\right)
$$

where $\omega_{i}$ is any 2-form generating $H^{2}\left(\mathbb{R}^{3} \backslash\left\{p_{i}\right\}\right)$ with the normalisation $\int_{\partial B_{r}\left(p_{i}\right)} \omega_{i}=$ $\delta_{i j}$ (for sufficiently small $r$ ) and $\eta_{i}$ is any form verifying $d \eta_{i}=u^{*} \omega_{i}$.

Proof of proposition 2.3. We first replace $S^{4}$ with $\mathbb{R}^{4}$. If $\bar{\omega}_{i}$ denotes the unit volume-form on $S_{i}^{2}$, we take $\omega_{i}=\frac{1}{4 \pi} \pi_{i}^{*} \bar{\omega}_{i}$ thus $u^{*} \omega_{i}=\frac{1}{4 \pi} u_{i}^{*} \bar{\omega}_{i}$. We also take a particular choice for $\eta_{i}$ such that $d \eta_{i}=u^{*} \omega_{i}$ : Having in mind that $-\frac{1}{4 \pi^{2}} \frac{1}{|x|^{2}}$ is the green kernel of the Laplacian on $\mathbb{R}^{4}$, we take

$$
\eta_{i}=d^{*}\left[-\frac{1}{4 \pi^{2}} \frac{1}{|x|^{2}} \star u^{*} \omega_{i}\right]
$$

If we explicit the convolution $\star$ we get

$$
\begin{aligned}
& \eta_{i}=-\frac{1}{4 \pi^{2}} d^{*}\left[\sum_{k<l} d x_{k} d x_{l} \int_{\mathbb{R}^{4}} \frac{\Omega_{\mathbb{R}^{4}}}{|x-y|^{2}}\left(u^{*} \omega_{i}, d y_{k} d y_{l}\right)\right] \\
& =\frac{1}{4 \pi^{2}} \sum_{k<l} \sum_{j=1}^{4} *\left(d x_{j} *\left(d x_{k} d x_{l}\right)\right) \int_{\mathbb{R}^{4}} \frac{x_{j}-y_{j}}{|x-y|^{4}} *\left(d y_{k} d y_{l}\right) \wedge u^{*} \omega_{i}(y) \\
& =\frac{1}{4 \pi^{2}} \sum_{k \neq l} d x_{k} \int_{\mathbb{R}^{4}} \frac{x_{l}-y_{l}}{|x-y|^{4}} *\left(d y_{k} d y_{l}\right) \wedge u^{*} \omega_{i}(y)
\end{aligned}
$$


Substituting in the formula for $N(u)$ we get

$$
\begin{aligned}
& \int_{S^{4}} u^{*} \omega_{1} \wedge \eta_{1} \wedge \eta_{2} \\
& =\frac{1}{16 \pi^{4}} \sum_{i \neq k} \sum_{j \neq l} \int_{\left(\mathbb{R}^{4}\right)^{3}} \frac{x_{i}-y_{i}}{|x-y|^{4}} \frac{x_{j}-y_{j}}{|x-y|^{4}} \\
& \quad u^{*} \omega_{1}(x) \wedge d x_{k} d x_{l} \wedge u^{*} \omega_{1}(y) \wedge *\left(d y_{i} d y_{k}\right) \wedge u^{*} \omega_{2}(z) \wedge *\left(d z_{j} d z_{l}\right)
\end{aligned}
$$

The coarea formula of Federer tells us that $\forall F: \mathbb{R}^{4} \rightarrow S^{2}$ and any 2 -form $\alpha$ in $\mathbb{R}^{4}$ we have

$$
\int_{\mathbb{R}^{4}} \alpha \wedge F^{*} \Omega_{S^{2}}=\int_{\xi \in S^{2}} \Omega_{S^{2}}(\xi) \int_{F^{-1}(\xi)} \alpha
$$

Where $\Omega_{S^{2}}$ is the volume form on $S^{2}$. Applying the coarea formula three times in (2.7), writing $u^{*} \omega_{i}=u^{*} \bar{\omega}_{i} / 4 \pi$ we obtain, using proposition 2.1 ,

$$
\begin{aligned}
& \int_{S^{4}} u^{*} \omega_{1} \wedge \eta_{1} \wedge \eta_{2} \\
& =\frac{1}{\left|S^{2}\right|^{3}} \int_{\left(S_{1}^{2}\right)^{2} \times S_{2}^{2}} \operatorname{rlk}\left(u_{1}^{-1}(\xi) \mid u_{1}^{-1}(\zeta), u_{2}^{-1}(\nu)\right) \omega_{1}(\xi) \wedge \omega_{1}(\zeta) \wedge \omega_{2}(\nu)
\end{aligned}
$$

Now observe that the integral $\int_{S^{4}} u^{*} \omega_{1} \wedge \eta_{1} \wedge \eta_{2}$ is independent on the choices of the generators of $H^{2}\left(S^{2}\right)$ and is also independent on the choice of the $\eta_{i}$ such that $d \eta_{i}=u^{*} \omega_{i}$.

Take $\xi_{0}$ and $\zeta_{0}$ two regular points of $u_{1}$ and $\nu_{0}$ a regular point of $u_{2}$. Replace now in the arguments above $\bar{\omega}_{1}(\xi), \bar{\omega}_{1}(\zeta)$ and $\bar{\omega}_{2}(\nu)$ respectively by $\tilde{\omega}_{1}^{\xi_{0}}(\xi)=$ $\frac{1}{\left|B_{\sigma}\left(\xi_{0}\right)\right|} \chi_{B_{\sigma}\left(\xi_{0}\right)} \bar{\omega}_{1}(\xi) \tilde{\omega}_{1}^{\zeta_{0}}(\zeta)=\frac{1}{\left|B_{\sigma}\left(\zeta_{0}\right)\right|} \chi_{B_{\sigma}\left(\zeta_{0}\right)} \bar{\omega}_{1}(\zeta)$ and $\tilde{\omega}_{2}^{\nu_{0}}(\nu)=\frac{1}{\left|B_{\sigma}\left(\nu_{0}\right)\right|} \chi_{B_{\sigma}\left(\nu_{0}\right)} \bar{\omega}_{2}(\nu)$, where $B_{\sigma}$ denotes a geodesic ball in $S^{2}$ of radius $\sigma$ and $\chi_{B_{\sigma}}$ is the characteristic function of this geodesic balls. We choose $\sigma$ sufficiently small such that $\operatorname{rlk}\left(u_{1}^{-1}(\xi) \mid u_{1}^{-1}(\zeta), u_{2}^{-1}(\nu)\right)$ is independent of the choice of the triplet in $B_{\sigma}\left(\xi_{0}\right) \times$ $B_{\sigma}\left(\zeta_{0}\right) \times B_{\sigma}\left(\nu_{0}\right)$ and then equal to $\operatorname{rk}\left(u_{1}^{-1}\left(\xi_{0}\right) \mid u_{1}^{-1}\left(\zeta_{0}\right), u_{2}^{-1}\left(\nu_{0}\right)\right)$. So arguing like above we obtain.

$$
\int_{S^{4}} u^{*} \omega_{1} \wedge \eta_{1} \wedge \eta_{2}=\operatorname{rlk}\left(u_{1}^{-1}\left(\xi_{0}\right) \mid u_{1}^{-1}\left(\zeta_{0}\right), u_{2}^{-1}\left(\nu_{0}\right)\right)
$$

this proves proposition 2.3 .

2.3. Systems of "small caps". we need the following elementary lemma.

Lemma 2.1. Let $d A$ and $d B$ be two 2-forms of $S^{4}$ such that

$$
d A \wedge d A=0 \quad \text { and } \quad d B \wedge d B=0 \quad \text { in } S^{4}
$$

assume they both define laminations $\mathcal{L}^{A}$ and $\mathcal{L}^{B}$. If they commute :

$$
d A \wedge d B=0
$$

then for $d A$-a.e. $x$ and for $d B$-a.e. $y$

$$
\mathcal{L}^{A}(x) \cap \mathcal{L}^{B}(y)=\emptyset
$$


Proof of lemma 2.1. Assume this is not the case, then we may find two saturated subsets $\mathcal{S}^{A}$ and $\mathcal{S}^{B}$ of $\mathcal{L}^{A}$ and $\mathcal{L}^{B}$ of non zero $d A$ and $d B$ measures such that $\forall x \in \mathcal{S}^{A}$ and $\forall y \in \mathcal{S}^{B}$

$$
\mathcal{L}^{A}(x) \cap \mathcal{L}^{B}(y) \neq \emptyset
$$

Because of the lamination hypothesis, we may also take $\mathcal{S}^{A}$ and $\mathcal{S}^{B}$ to be subparts of $S^{4}$ where respectively $d A$ and $d B$ do not degenerate with $|d A| \geq c>0$ on $\mathcal{S}^{A}$ and $|d B| \geq c>0$ on $\mathcal{S}^{B}$. Then, since $d A \wedge d B=0, d B$ restricted to $\mathcal{L}^{A}(x)$ defines a 1-foliation. Then there exists an at most countable union of 1-dimensional curves $\left(\Gamma_{n}\right)_{n \in \mathbb{N}}$ in $\mathcal{L}^{A}(x)$ transverse to this foliation such that every leaf of this 1-dimensional foliation crosses $\Gamma=\cup_{n} \Gamma_{n}$. Since every $d B$-leaf of $\mathcal{S}^{B}$ is assumed to intersect $\mathcal{L}^{A}(x)$, every $d B$-leaf of $\mathcal{S}^{B}$ intersects $\Gamma=\cup_{n} \Gamma_{n}$ that has $d B$-measure 0 . So $\mathcal{S}^{B}$ has $d B-$ measure 0 which is a contradiction.

Proposition-Definition 2.1. Let $d A_{1}, d A_{2}$ and $d A_{3}$ be 3 closed 2-forms on $S^{4}$ such that

$$
\forall i, j=1,2,3 \quad d A_{i} \wedge d A_{j}=0
$$

Assume that the $d A_{i}$ define laminations. Denote by $\mathcal{L}^{A_{i}}(x)$ the leaf of $d A_{i}$ that passes through $x$. Then for $d A_{1} \times d A_{2} \times d A_{3}-$ a.e. leaves $\mathcal{L}^{A_{1}}\left(x_{1}\right) \times \mathcal{L}^{A_{2}}\left(x_{2}\right) \times \mathcal{L}^{A_{3}}\left(x_{3}\right)$ the following property holds : for any triplet of subsets $K_{1}, K_{2}$ and $K_{3}$ of $\mathcal{L}^{A_{1}}\left(x_{1}\right)$, $\mathcal{L}^{A_{2}}\left(x_{2}\right)$ and $\mathcal{L}^{A_{3}}\left(x_{3}\right)$ such that the curvatures of $\partial K_{1}, \partial K_{2}$ and $\partial K_{3}$ are uniformly bounded we may assign 3 union of embedded 2-disks $C_{K_{1}}, C_{K_{2}}$ and $C_{K_{3}}$ verifying

$$
\begin{gathered}
\forall i=1,2,3 \quad\left|C_{K_{i}}\right| \leq C\left|\partial K_{i}\right| \\
\left|\int_{C_{K_{1}}} \int_{C_{K_{2}}} \int_{C_{K_{3}}} \mathcal{G}\right| \leq C\left|\partial K_{1}\right|\left|\partial K_{2}\right|\left|\partial K_{3}\right|
\end{gathered}
$$

- $\forall i=1,2,3$

$$
\left|\int_{K_{i}} \int_{C_{K_{i+1}}} \int_{C_{K_{i-1}}} \mathcal{G}\right| \leq C\left|K_{i}\right|\left|\partial K_{i+1}\right|\left|\partial K_{i-1}\right|
$$

- $\forall i=1,2,3$

$$
\left|\int_{K_{i}} \int_{K_{i+1}} \int_{C_{K_{i-1}}} \mathcal{G}\right| \leq C\left|K_{i}\right|\left|K_{i+1}\right|\left|\partial K_{i-1}\right|
$$

where $\mathcal{G}$ is the Gauss-form given by (2.2). where the constant $C$ may depend on the $x_{i}$ but not on the $K_{i}$ in $\mathcal{L}^{A_{i}}\left(x_{i}\right)$. A choice of such an assignment is called a system of "small caps".

Proof of proposition 2.1. For convenience of the presentation we give the proof in the case where the $d A_{i}$ 's are supported in a compact sub-domain of $\mathbb{R}^{4}$. Because of the previous lemma, for $d A_{1} \times d A_{2} \times d A_{3}$ a.e. triplet $\left(x_{1}, x_{2}, x_{3}\right) \forall i \neq j, \mathcal{L}^{A_{i}}\left(x_{i}\right) \cap$ $\mathcal{L}^{A_{j}}\left(x_{j}\right)=\emptyset$. Moreover since they define Laminations, for $d A_{1} \times d A_{2} \times d A_{3}$ a.e. 
triplets $\left(x_{1}, x_{2}, x_{3}\right)\left|d A_{i}\right| \geq c>0$ on $\mathcal{L}^{A_{i}}\left(x_{i}\right)$ for $i=1,2,3$ and then the three leaves $\mathcal{L}^{A_{i}}\left(x_{i}\right)$ for $i=1,2,3$ have bounded geometries. Take such a triplet. We then may find a Lipschitz diffeomorphism of $\mathbb{R}^{4}$ such that the restriction to the 3 skeleton of a given lattice $L_{\delta}=\delta \mathbb{Z}^{4}$ of $\mathbb{R}^{4}$ (for a sufficiently small size $\delta$ ) of the 3 leaves are made of flat segments. Since all of the $\partial K_{i}$ have uniformly bounded curvature, we may modify $K_{i}$ a bit, keeping it's area proportional to the original one and the length of $\partial K_{i}$ proportional to it's original one, in order to ensure that $\partial K_{i}$ lies in the 3 -skeleton of $L_{\delta}$ keeping $K_{i}$ in it's leaf $\mathcal{L}^{A_{i}}\left(x_{i}\right)$ and also to ensure that $\partial K_{i}$ is made of a union of straight segments such that each connected component of $\partial K_{i}$ restricted to any 3 -cell is made exactly of 1 segment. Take now one component of $\partial K_{i}$ denote it by $l_{i}$. $l_{i}$ admits a projection $\hat{l}_{i}$ in the 1 -skeleton of $\delta \mathbb{Z}^{4}$ such that the area of the annulus $a_{i}$ bounding $l_{i} \cup \hat{l}_{i}$ is proportional to the length of $l_{i}$, moreover $\left|l_{i}\right| \simeq\left|\hat{l}_{i}\right|$. Solve now the plateau problem for $\hat{l}_{i}$ and denote by $d_{i}$ a minimal disk that bounds $\hat{l}_{i}$. Since $\hat{l}_{i}$ lies in a compact part of $\mathbb{R}^{4}$ we clearly have $\left|d_{i}\right| \leq C\left|\hat{l}_{i}\right|$. We project now $d_{i}$ in the 2-skeleton of $L_{\delta}$ in the following way : first we project $d_{i}$ in the 3-skeleton using the following argument. Let $c_{\delta}$ be a given 4-cell of $L_{\delta}$, we claim that we can choose a point $p$ in the interior of $c_{\delta}$ such that the radial projection $\pi_{p}$ from $c_{\delta}$ onto $\partial c_{\delta}$ relative to $p$ keeps the area of the projection of $d_{i} \cap c_{\delta}$ proportional to the area of $d_{i} \cap c_{\delta}$ itself. Indeed let $v$ be the conformal map from the unit disk solving our Plateau problem for $\hat{l}_{i}$, we have

$$
\operatorname{area}\left(c_{\delta} \cap d_{i}\right)=\frac{1}{2} \int_{v^{-1}\left(c_{\delta} \cap d_{i}\right)}|\nabla v(x)|^{2} d x
$$

Simple computations show that

$$
\operatorname{area}\left(\pi_{p}\left(c_{\delta} \cap d_{i}\right)\right) \leq C \int_{v^{-1}\left(c_{\delta} \cap d_{i}\right)} \frac{|\nabla v|^{2}}{|v(x)-p|^{2}} d x
$$

integrating over $p$ in the half 4 -cube $c_{\delta} / 2$ we get

$$
\begin{aligned}
& \int_{p \in c_{\delta} / 2} \operatorname{area}\left(\pi_{p}\left(c_{\delta} \cap d_{i}\right)\right) d p \leq C \int_{p \in c_{\delta} / 2} \int_{v^{-1}\left(c_{\delta} \cap d_{i}\right)} \frac{|\nabla v|^{2}}{|v(x)-p|^{2}} d x d p \\
& \leq C \int_{v^{-1}\left(c_{\delta} \cap d_{i}\right)}|\nabla v|^{2}
\end{aligned}
$$

applying the mean-value formula we get such a $p$. Then using the same idea we can project $\pi_{p}\left(c_{\delta} \cap d_{i}\right)$ onto the 2-cell of $L_{\delta}$ and we then obtain a disk $\hat{d}_{i}$ that bounds $\hat{l}_{i}$ and which is made of flat pieces of the 2-skeleton of $L_{\delta}$ and such that the number of pieces is bounded by $C\left|l_{i}\right| / \delta$. We take $C_{K_{i}}$ to be the union of these disks for the various components of $\partial K_{i}$. The reason why ii), iii) and iv) hold comes from the fact that the integral of the Gauss-form over a triplet of 2-parralelograms of size $\delta$ is bounded independently from their relative position in 4-space by a constant depending on $\delta$.

2.4. Leaf harmonic measures and ergodic theorem for leaf-heat diffusions on laminations. First of all we prove the following key observation.

Proposition 2.4. Let $d A$ be a closed 2-form of $S^{4}$ that is integrable : $d A \wedge d A=$ 0 . Assume that $d A$ defines a lamination $\mathcal{L}^{A}$. Denote by $\Omega_{S^{4}}$ the standard volume-form on $S^{4}$. Take the metric on $S^{4}$ to be $g^{A}=g_{S^{4}} /|d A|$. Then $\Omega_{S^{4}}$ is $g^{A}$-leaf-harmonic 
for the lamination defined by $d A$ :

$$
\begin{aligned}
& \forall \phi \in C^{\infty}\left(S^{4}\right) \quad \forall \mathcal{S} \quad \text { measurable non deg. saturated set of } \mathcal{L}^{A} \\
& \int_{\mathcal{S}} \Delta_{\mathcal{L}^{A}} \phi \Omega_{S^{4}}=0
\end{aligned}
$$

where $\Delta_{\mathcal{L}^{A}}$ denotes the Laplace-Beltrami operator restricted to the leaves of $\mathcal{L}^{A}$.

Proof of proposition 2.4. Let $\mathcal{S}$ be a non degenerated measurable saturated set : $|d A| \geq c>0$ on $\mathcal{S}$, for every $x \in \mathcal{S} \mathcal{L}^{A}(x) \subset \mathcal{S}$. Take a $\phi$ in $C^{\infty}\left(S^{4}\right)$, we may always assume that $\phi$ has a support contained in a sufficiently small ball $B_{r}(x)$ of center $x$ such that we can apply Darboux Theorem in the whole ball and we have on $B_{r}(x)$ $d A=H^{*} d x_{1} \wedge d x_{2}$ where $H$ is a map from $B_{r}(x)$ into $D^{2}$ (indeed since $d A$ defines a lamination, $\mathcal{S}$ is contained in a compact set where $|d A|>0$ and we can extract a finite covering from any covering of balls where Darboux theorem applies for $d A$ and construct a partition of unity from this covering). Applying the Coarea Formula to $H$ we have

$$
\begin{aligned}
\int_{\mathcal{S}} \Delta_{\mathcal{L}^{A}} \phi \Omega_{S^{4}} & =\int_{\xi \in D^{2} \cap H(\mathcal{S})} \int_{H^{-1}(\xi)} \Delta_{\mathcal{L}^{A} \phi} \frac{d \mathcal{H}^{2}}{|d A|} \\
& =\int_{\xi \in D^{2} \cap H(\mathcal{S})} \int_{H^{-1}(\xi)} \Delta_{\mathcal{L}^{A} \phi d \operatorname{vol}_{\mathcal{L}^{A}}}
\end{aligned}
$$

where $d \mathrm{vol}_{\mathcal{L}^{A}}$ is the volume form on the leaves induced by the metric $g_{S^{4}} /|d A|$. (Observe that $H^{-1}(\xi)$ is a portion of leaf from our lamination). So clearly

$$
\int_{H^{-1}(\xi)} \Delta_{\mathcal{L}^{A}} \phi d \operatorname{vol}_{\mathcal{L}^{A}}=0
$$

and the proposition 2.4 is proved.

Denote by $p^{A}(x, y, t)$ the heat-kernel for the Laplace Beltrami operator $\Delta_{\mathcal{L}^{A}}$ on the leaf $\mathcal{L}^{A}(x)$. Since the leaf is contained on a compact set where $|d A|>0$ it has bounded geometry for the metric induced by $g_{S^{4}} /|d A|$. So the leaf is complete for the diffusion and from [4] we have

$$
p^{A}(x, y, t)>0 \quad \text { and } \int_{\mathcal{L}^{A}(x)} p^{A}(x, y, t) d \operatorname{vol}_{\mathcal{L}^{A}(x)}(y)=1
$$

$p^{A}(x, d y, t)=p^{A}(x, y, t) d \operatorname{vol}_{\mathcal{L}^{A}}(y)$ defines a probability measure in $S^{4}$ in the following way

$$
p^{A}(x, E, t)=\int_{E \cap \mathcal{L}^{A}(x)} p^{A}(x, y, t) d \operatorname{vol}_{\mathcal{L}}(y)
$$

This probability measure verifies the Chapman-Kolmogorov equation

$$
p^{A}(t+s, x, E)=\int_{S^{4}} p^{A}(x, d y, t) p^{A}(y, E, s)
$$

We claim now that the standard volume form on $S^{4}$ is invariant under this diffusion : for any measurable non degenerate saturated set $\mathcal{S}$ we have

$$
\forall E \text { measurable } \quad \int_{\mathcal{S}} p^{A}(x, E, t) \Omega_{S^{4}}(x)=\int_{\mathcal{S} \cap E} \Omega_{S^{4}}
$$


or equivalently for any $f$ measurable in $\mathcal{S}$

$$
\int_{\mathcal{S}} \Omega_{S^{4}}(x) \int_{\mathcal{S}} p^{A}(x, y, t) f(y) d \operatorname{vol}_{\mathcal{L}^{A}}(y)=\int_{\mathcal{S}} f(x) \Omega_{S^{4}}(x)
$$

This result can be deduced from the theorem of L. Gardnett in [9] which says that being leaf harmonic for a measure implies it's invariance under the diffusion of the corresponding heat kernel. Formally the proof can be sketched like this : for any $f \in C^{\infty}$ we have, using the definition of the heat Kernel

$$
\left[\frac{\partial}{\partial t}+\Delta_{\mathcal{L}}\right]\left(\int_{\mathcal{S}} p^{A}(x, y, t) f(y) d \operatorname{vol}_{\mathcal{L}}(y)\right)=0
$$

Using the fact that $\Omega_{S^{4}}$ is leaf harmonic, taking $v$ to bev $=\int_{\mathcal{S}} p^{A}(x, y, t) f(y) d \operatorname{vol}_{\mathcal{L}}(y)$ and the characterization of leaf harmonicity of $\Omega_{S^{4}}$ applied to that $v$ (proposition 2.4) yields

$$
\int_{\mathcal{S}} \Omega_{S^{4}} \Delta_{\mathcal{L}^{A}}\left(\int_{\mathcal{S}} p^{A}(x, y, t) f(y) d \operatorname{vol}_{\mathcal{L}}^{A}(y)\right)=0
$$

Combining the two previous identities we obtain

$$
\frac{d}{d t}\left[\int_{\mathcal{S}} \Omega_{S^{4}}\left(\int_{\mathcal{S}} p^{A}(x, y, t) f(y) d \operatorname{vol}_{\mathcal{L}}(y)\right)\right]=0
$$

and since $\int_{\mathcal{S}} p^{A}(x, y, 0) f(y) d \operatorname{vol}_{\mathcal{L}}(y)=f$ we get that $\Omega_{S^{4}}$ is invariant under the diffusion.

Thus $p^{A}(x, E, t)$ defines a Markov process with the $\Omega_{S^{4}}$ measure as an invariant measure. Adapting the result by Yosida (see [21]) to our situation we deduce the following ergodic theorem

THEOREM 2.1. Let $d A$ be a closed integrable 2-form on $S^{4}$ (i.e. $d A \wedge d A=0$ ). Assume that $d A$ defines a lamination. Denote by $\mathcal{S}$ a measurable saturated set for $d A$ and by $p^{A}(x, y, t)$ the heat-kernel for the metric induced by $g_{S^{4}} /|d A|$ on every leaf of $d A\left(g_{S^{4}}\right.$ is the standard metric on $\left.S^{4}\right)$. Then for any $f$ in $L^{1}\left(\mathcal{S} ; \Omega_{S^{4}}\right)$ the following limit exists almost everywhere

$$
\lim _{T \rightarrow+\infty} \frac{1}{T} \int_{0}^{T} d t \int_{\mathcal{S}} f(y) p^{A}(x, d y, t)=f_{\star}
$$

and is in $L^{1}$. Moreover we have

$$
\int_{\mathcal{S}} f_{\star} \Omega_{S^{4}}=\int_{\mathcal{S}} f \Omega_{S^{4}}
$$

3. Proof of theorem 1.1. Let $d A$ and $d B$ be two integrable closed 2-forms of $S^{4}$ which commute $(d A \wedge d B=0)$. and assume that they both define laminations : the zero sets of $d A$ and $d B$ are respectively $d A$ and $d B$-negligeable. For the convenience of the presentation we assume that both $d A$ and $d B$ are defined on a compact subset of $\mathbb{R}^{4}$. From the lamination hypothesis it is not difficult to deduce the existence of saturated subsets $\mathcal{S}_{\varepsilon}^{A}$ and $\mathcal{S}_{\varepsilon}^{B}$ for respectively $d A$ and $d B$ such that

- $|d A| \geq c_{\varepsilon}>0$ on $\mathcal{S}_{\varepsilon}^{A}$ and $|d B| \geq c_{\varepsilon}>0$ on $\mathcal{S}_{\varepsilon}^{B}$. 


$$
\left|\int_{\mathcal{S}_{\varepsilon}^{A} \times \mathcal{S}_{\varepsilon}^{A} \times \mathcal{S}_{\varepsilon}^{B}} \mathcal{G}^{A, A, B}(x, y, z)-\int_{\mathbb{R}^{4} \times \mathbb{R}^{4} \times \mathbb{R}^{4}} \mathcal{G}^{A, A, B}(x, y, z)\right| \leq \varepsilon
$$

where

$$
\begin{aligned}
& \mathcal{G}^{A, A, B}(x, y, z) \\
& =\frac{1}{4 \pi^{4}} \sum_{i \neq k} \sum_{j \neq l} \frac{x_{i}-y_{i}}{|x-y|^{4}} \frac{x_{j}-y_{j}}{|x-y|^{4}} \\
& \quad d A(x) \wedge d x_{k} d x_{l} \wedge d A(y) \wedge *\left(d y_{i} d y_{k}\right) \wedge d B(z) \wedge *\left(d z_{j} d z_{l}\right)
\end{aligned}
$$

Using theorem 2.1 we know the existence of the limit

$$
\begin{aligned}
& \lim _{T \rightarrow+\infty} \frac{1}{T^{3}} \int_{[0, T]^{3}} \int_{\mathcal{S}_{\varepsilon}^{A, A, B}} p^{A}(x, d \xi, t) p^{A}(y, d \zeta, s) p^{B}(z, d \nu, \sigma) g^{A A B}(\xi, \zeta, \nu) \\
& =\Lambda^{\star}(x, y, z)
\end{aligned}
$$

for a.e. $(x, y, z) \in \mathcal{S}_{\varepsilon}^{A} \times \mathcal{S}_{\varepsilon}^{A} \times \mathcal{S}_{\varepsilon}^{B}$ where

$$
g^{A A B}(\xi, \zeta, \nu)=\left\langle\mathcal{G}^{A, A, B} ; \Omega_{\mathbb{R}^{4}}^{3}\right\rangle(\xi, \zeta, \nu)
$$

and that

$$
\int_{\mathcal{S}_{\varepsilon}^{A, A, B}} \Lambda^{\star} \Omega_{\mathbb{R}^{4}}^{3}=\int_{\mathcal{S}_{\varepsilon}^{A, A, B}} \mathcal{G}^{A, A, B}
$$

where $\mathcal{S}_{\varepsilon}^{A, A, B}$ denotes $\mathcal{S}_{\varepsilon}^{A} \times \mathcal{S}_{\varepsilon}^{A} \times \mathcal{S}_{\varepsilon}^{B}$, and $p^{A}$ and $p^{B}$ are respectively the heat kernels for the heat operator on the leaves of $d A$ and $d B$ for the metrics induced by $g_{\mathbb{R}^{4}} /|d A|$ and $g_{\mathbb{R}^{4}} /|d B|$. Moreover $p^{A}(x, d \xi, t)$ denotes the distribution form $p^{A}(x, \xi, t) d \operatorname{vol}_{\mathcal{L}^{A}(x)}(\xi)$ and $d \operatorname{vol}_{\mathcal{L}^{A}(x)}$ is the volume form of the $d A$-leaf passing by $x$ induced by $g_{\mathbb{R}^{4}} /|d A|$ whose associated measure on the leaf is $d \mathcal{H}^{2} /|d A|\left\lfloor\mathcal{L}^{A}\left(d \mathcal{H}^{2}\right.\right.$ is the two-dimensional Hausdorff measure). We modify $p^{A}$ and $p^{B}$ in the following way. Let $\delta_{T}>0$ to be fixed later on in the proof, we just assume now that $\delta_{T} \rightarrow 1$ as $T \rightarrow+\infty$ and we omit the subscript $T$. Using the mean value formula we get that for every integer $k$ there exists $\delta^{k+1}<p<\delta^{k}$ such that

$$
\begin{aligned}
& \left|\left\{p^{A}(x, \xi, t)=p\right\}\right|=\int_{\left\{\xi ; p^{A}(x, \xi, t)=p\right\}} \frac{d \mathcal{H}^{1}}{|d A|^{\frac{1}{2}}(\xi)} \\
& \leq \frac{1}{1-\delta} \frac{1}{\delta^{k}} \int_{\delta^{k+1}}^{\delta^{k}} d q \int_{\left\{\xi ; p^{A}(x, \xi, t)=q\right\}} \frac{d \mathcal{H}^{1}}{|d A|^{\frac{1}{2}}(\xi)} \\
& \leq \frac{1}{1-\delta} \frac{1}{\delta^{k}} \int_{\mathcal{L}^{A}(x) \cap\left\{\xi ; \delta^{k+1} \leq p^{A}(x, \xi, t) \leq \delta^{k}\right\}}\left|\nabla_{\xi} p\right| d \operatorname{vol}_{\mathcal{L}^{A}(x)}(\xi)
\end{aligned}
$$

where we used the coarea formula. Choose such a $p$, denote it by $p_{k, \delta}^{A}(x, t)$ and let

$$
\mathcal{L}_{k, \delta}^{A}(x, t)=\left\{\xi \in \mathcal{L}^{A}(x) \quad \text { s. t. } \quad p_{k+1, \delta}^{A}(x, t) \leq p^{A}(x, \xi, t) \leq p_{k, \delta}^{A}(x, t)\right\}
$$


Denote by $\tilde{p}^{A}(x, \xi, t)$ the approximated kernel such that

$$
\tilde{p}^{A}(x, \xi, t)=p_{k, \delta}^{A}(x, t) \quad \text { on } \quad \mathcal{L}_{k, \delta}^{A}(x, t)
$$

By construction we have

$$
\forall \xi \in \mathcal{L}^{A}(x) \quad \forall t>0 \quad\left|\frac{p^{A}(x, \xi, t)-\tilde{p}^{A}(x, \xi, t)}{p^{A}(x, \xi, t)}\right| \leq 1-\frac{1}{\delta}
$$

keeping in mind that $\delta$ tends to 1 as $T \rightarrow+\infty$ so that the relative difference between $p^{A}$ and $\tilde{p}^{A}$ will be small. Precisely we have, omitting to explicitly write $x, y, z$ and $t$, $s, \sigma$,

$$
\begin{aligned}
& p^{A}(d \xi)=\tilde{p}^{A}(d \xi)+\left[\frac{p^{A}-\tilde{p}^{A}}{p^{A}}\right](\xi) p^{A}(d \xi) \\
& p^{A}(d \zeta)=\tilde{p}^{A}(d \zeta)+\left[\frac{p^{A}-\tilde{p}^{A}}{p^{A}}\right](\zeta) p^{A}(d \zeta)
\end{aligned}
$$

and

$$
p^{B}(d \nu)=\tilde{p}^{B}(d \nu)+\left[\frac{p^{B}-\tilde{p}^{B}}{p^{B}}\right](\nu) p^{B}(d \nu)
$$

Clearly, from (3.5) we deduce

$$
\begin{aligned}
& \int_{\mathcal{S}_{\varepsilon}^{A, A, B}}\left|\int_{\mathcal{S}_{\varepsilon}^{A, A, B}}\left[\frac{\tilde{p}^{A}-p^{A}}{p^{A}}\right] g^{A A B} p^{A}(d \xi) p^{A}(d \zeta) p^{B}(d \nu)\right| \\
& \leq\left(1-\frac{1}{\delta}\right) \int_{\mathcal{S}_{\varepsilon}^{A, A, B}} \int_{\mathcal{S}_{\varepsilon}^{A, A, B}} p^{A}(d \xi) p^{A}(d \zeta) p^{B}(d \nu) \frac{|d A|(\xi)|d A|(\zeta)|d B|(\nu)}{|\xi-\zeta|^{3}|\xi-\nu|^{3}}
\end{aligned}
$$

Taking the time average of it and the limit as $T \rightarrow+\infty$, we have

$$
\begin{aligned}
& \lim _{T \rightarrow+\infty} \int_{\mathcal{S}_{\varepsilon}^{A}, A, B} \frac{1}{T^{3}} \int_{[0, T]^{3}}\left|\int_{\mathcal{S}_{\varepsilon}^{A, A, B}}\left[\frac{\tilde{p}^{A}-p^{A}}{p^{A}}\right](\xi) p^{A}(d \xi) p^{A}(d \zeta) p^{B}(d \nu) g^{A A B}\right| \\
& \leq \lim _{T \rightarrow+\infty}\left(1-\frac{1}{\delta_{T}}\right) \int_{\mathcal{S}_{\varepsilon}^{A, A, B}} \frac{|d A|(x)|d A|(y)|d B|(z)}{|x-y|^{3}|x-z|^{3}}=0
\end{aligned}
$$

Thus for a.e. $(x, y, z)$ in $S_{\varepsilon}^{A, A, B}$ we have

$$
\lim _{T \rightarrow+\infty} \frac{1}{T^{3}} \int_{[0, T]^{3}} \int_{\mathcal{S}_{\varepsilon}^{A, A, B}}\left[\frac{\tilde{p}^{A}-p^{A}}{p^{A}}\right](\xi) p^{A}(d \xi) p^{A}(d \zeta) p^{B}(d \nu) g^{A A B}=0
$$

This result can be extended in a similar way to all the other error terms when we replace $p$ by $\tilde{p}$ in (3.2), so that we obtain for a.e. $(x, y, z)$ in $S_{\varepsilon}^{A, A, B}$

$$
\begin{aligned}
& \lim _{T \rightarrow+\infty} \frac{1}{T^{3}} \int_{[0, T]^{3}} \int_{\mathcal{S}_{\varepsilon}^{A, A, B}} \tilde{p}^{A}(x, d \xi, t) \tilde{p}^{A}(y, d \zeta, s) \tilde{p}^{B}(z, d \nu, \sigma) g^{A A B}(\xi, \zeta, \nu) \\
& =\Lambda^{\star}(x, y, z)
\end{aligned}
$$


and

$$
\int_{\mathcal{S}_{\varepsilon}^{A, A, B}} \mathcal{G}^{A, A, B}=\int_{\mathcal{S}_{\varepsilon}^{A, A, B}} \lim _{T \rightarrow+\infty} \frac{1}{T^{3}} \int_{[0, T]^{3}} \int_{\mathcal{S}_{\varepsilon}^{A, A, B}} g^{A A B} \tilde{p}^{A}(d \xi) \tilde{p}^{A}(d \zeta) \tilde{p}^{B}(d \nu)
$$

We decompose now the leaves along the sets $\mathcal{L}_{k, \delta}^{A}, \mathcal{L}_{l, \delta}^{A}$ and $\mathcal{L}_{m, \delta}^{B}$ where the $\tilde{p}^{A}$ and $\tilde{p}^{B}$ are constant, and we obtain

$$
\begin{aligned}
& \int_{\mathcal{S}_{\varepsilon}^{A, A, B}} g^{A A B} \tilde{p}^{A}(d \xi) \tilde{p}^{A}(d \zeta) \tilde{p}^{B}(d \nu)= \\
& \sum_{k, l, m} \delta^{k+l+m} \int_{\mathcal{L}_{k, \delta}^{A}(x, t)} \int_{\mathcal{L}_{l, \delta}^{A}(y, s)} \int_{\mathcal{L}_{m, \delta}^{B}(z, \sigma)} g^{A A B} d v o l_{\mathcal{L}^{A}(x)} d v o l_{\mathcal{L}^{A}(y)} d v o l_{\mathcal{L}^{B}(z)}
\end{aligned}
$$

Recall that the volumes are taken with respect to the metrics $g^{A}=g_{\mathbb{R}^{4}} /|d A|, g^{A}=$ $g_{\mathbb{R}^{4}} /|d A|$ and $g^{B}=g_{\mathbb{R}^{4}} /|d B|$. We claim now that the restriction of $\mathcal{G}^{A, A, B}$ to $\mathcal{L}^{A}(x) \times$ $\mathcal{L}^{A}(y) \times \mathcal{L}^{B}(z)$ coincide with $g^{A A B} d v l_{\mathcal{L}^{A}(x)} d v o l_{\mathcal{L}^{A}(y)} d v o l_{\mathcal{L}^{B}(z)}$. Indeed it suffices to observe that for any $i$ and $j$ in $\{1 \ldots 4\}$, we have

$$
\left|\left\langle d A \wedge d x_{i} d x_{j} ; \Omega_{\mathbb{R}^{4}}\right\rangle\right|=|d A|\left|i_{\mathcal{L}^{A}}^{*} d x_{i} d x_{j}\right|
$$

where $i_{\mathcal{L}^{A}}$ is the isometric embedding of $\mathcal{L}^{A}$ into $\mathbb{R}^{4}$ and | | denotes the scalar product on 2 -forms induced by the canonical scalar product in $\mathbb{R}^{4}, g_{\mathbb{R}^{4}}$. Taking into account the orientation and the fact that $g^{A}=g_{\mathbb{R}^{4}} /|d A|$ we have

$$
\left\langle d A \wedge d x_{i} d x_{j} ; \Omega_{\mathbb{R}^{4}}\right\rangle=\left\langle i_{\mathcal{L}^{A}}^{*} d x_{i} d x_{j} ; d \operatorname{vol}_{\mathcal{L}^{A}}\right\rangle
$$

So then

$$
\begin{aligned}
\left\langle d A \wedge d x_{i} d x_{j} ; \Omega_{\mathbb{R}^{4}}\right\rangle d v o l_{\mathcal{L}^{A}} & =\left\langle i_{\mathcal{L}^{A}}^{*} d x_{i} d x_{j} ; d v o l_{\mathcal{L}^{A}}\right\rangle d v o l_{\mathcal{L}^{A}} \\
& =i_{\mathcal{L}^{A}}^{*} d x_{i} d x_{j}
\end{aligned}
$$

Thus

$$
\begin{aligned}
& \int_{\mathcal{L}_{k, \delta}^{A}(x, t)} \int_{\mathcal{L}_{l, \delta}^{A}(y, s)} \int_{\mathcal{L}_{m, \delta}^{B}(z, \sigma)} g^{A A B} d v o l_{\mathcal{L}^{A}(x)} d v o l_{\mathcal{L}^{A}(y)} d v o l_{\mathcal{L}^{B}(z)} \\
& =\int_{\mathcal{L}_{k, \delta}^{A}(x, t) \times \mathcal{L}_{l, \delta}^{A}(y, s) \times \mathcal{L}_{m, \delta}^{B}(z, \sigma)} \mathcal{G}
\end{aligned}
$$

where $\mathcal{G}$ is the Gauss-form introduced in proposition 2.1. We need now to close the $\mathcal{L}_{k, \delta}^{A}(x, t)$, the $\mathcal{L}_{l, \delta}^{A}(y, s)$ and the $\mathcal{L}_{m, \delta}^{B}(z, \sigma)$ by the mean of the system of "small caps" introduced in proposition 2.1. Let $\mathcal{D}_{k, \delta}^{A}(x, t)=\mathcal{L}_{k, \delta}^{A}(x, t) \cup C_{\partial \mathcal{L}_{k, \delta}^{A}(x, t)}$...etc, where $C_{\partial \mathcal{L}_{k, \delta}^{A}(x, t)}$ is the union of small caps for the connected components of $\partial \mathcal{L}_{k, \delta}^{A}(x, t)$ 
given by proposition 2.1. Using the result in this proposition we deduce that

$$
\begin{aligned}
& \left|\int_{\mathcal{L}_{k, \delta}^{A}(x, t) \times \mathcal{L}_{l, \delta}^{A}(y, s) \times \mathcal{L}_{m, \delta}^{B}(z, \sigma)} \mathcal{G}-\int_{\mathcal{D}_{k, \delta}^{A}(x, t) \times \mathcal{D}_{l, \delta}^{A}(y, s) \times \mathcal{D}_{m, \delta}^{B}(z, \sigma)} \mathcal{G}\right| \leq \\
& \leq C\left|\partial \mathcal{L}_{k, \delta}^{A}(x, t)\right|\left|\mathcal{L}_{l, \delta}^{A}(y, s)\right|\left|\mathcal{L}_{m, \delta}^{B}(z, \sigma)\right|+\left|\mathcal{L}_{k, \delta}^{A}(x, t)\right|\left|\partial \mathcal{L}_{l, \delta}^{A}(y, s)\right|\left|\mathcal{L}_{m, \delta}^{B}(z, \sigma)\right| \\
& +C\left|\mathcal{L}_{k, \delta}^{A}(x, t)\right|\left|\mathcal{L}_{l, \delta}^{A}(y, s)\right|\left|\partial \mathcal{L}_{m, \delta}^{B}(z, \sigma)\right|+\left|\partial \mathcal{L}_{k, \delta}^{A}(x, t)\right|\left|\partial \mathcal{L}_{l, \delta}^{A}(y, s)\right|\left|\mathcal{L}_{m, \delta}^{B}(z, \sigma)\right| \\
& +C\left|\partial \mathcal{L}_{k, \delta}^{A}(x, t)\right|\left|\mathcal{L}_{l, \delta}^{A}(y, s)\right|\left|\partial \mathcal{L}_{m, \delta}^{B}(z, \sigma)\right|+\left|\mathcal{L}_{k, \delta}^{A}(x, t)\right|\left|\partial \mathcal{L}_{l, \delta}^{A}(y, s)\right|\left|\partial \mathcal{L}_{m, \delta}^{B}(z, \sigma)\right| \\
& +C\left|\partial \mathcal{L}_{k, \delta}^{A}(x, t)\right|\left|\partial \mathcal{L}_{l, \delta}^{A}(y, s)\right|\left|\partial \mathcal{L}_{m, \delta}^{B}(z, \sigma)\right|
\end{aligned}
$$

Multiplying the quantities in the right-hand side of (3.15) by $\delta^{k+l+m}$ and summing over $k, l$ and $m$, using (3.4), we get for the first term for instance

$$
\begin{aligned}
& \sum_{k, l, m} \delta^{k+l+m}\left|\partial \mathcal{L}_{k, \delta}^{A}(x, t)\right|\left|\mathcal{L}_{l, \delta}^{A}(y, s)\right|\left|\mathcal{L}_{m, \delta}^{B}(z, \sigma)\right| \\
& \leq \frac{1}{(1-\delta) \delta^{3}} \sum_{k, l, m} \int_{\tilde{\mathcal{L}}_{k, \delta}^{A}(x, t)}\left|\nabla_{\xi} p^{A}\right| \int_{\mathcal{L}_{l, \delta}^{A}(y, s)} p^{A}(y, d \zeta, s) \int_{\mathcal{L}_{m, \delta}^{B}(z, \sigma)} p^{B}(z, d \nu, \sigma) \\
& \leq \frac{1}{(1-\delta) \delta^{3}} \int_{\mathcal{L}^{A}(x)}\left|\nabla_{\xi} p^{A}\right| d v o l_{\mathcal{L}^{A}}
\end{aligned}
$$

where $\tilde{\mathcal{L}}_{k, \delta}^{A}(x, t)=\mathcal{L}^{A}(x) \cap\left\{\xi ; \delta^{k+1} \leq p^{A}(\xi) \leq \delta^{k}\right\}$ we have used the fact that $\int_{\mathcal{L}^{A}(y)} p^{A}=1$ and $\int_{\mathcal{L}^{B}(z)} p^{B}=1$.

This is now the step where the Liouville hypothesis on the lamination plays a crucial role :

We have

$$
\int_{\mathcal{L}^{A}}\left|\nabla_{\xi} p^{A}\right| d \operatorname{dvol}_{\mathcal{L}^{A}} \leq C\left(\int_{\mathcal{L}^{A}} p^{A}(x, \xi, t)\right)^{\frac{1}{2}}\left(\int_{\mathcal{L}^{A}} \frac{\left|\nabla_{\xi} p^{A}\right|^{2}}{p^{A}}\right)^{\frac{1}{2}}
$$

moreover a short computation shows that for $t>0$

$$
\frac{\partial\left(p^{A} \log p^{A}\right)}{\partial t}+\Delta\left(p^{A} \log p^{A}\right)=-\frac{\left|\nabla p^{A}\right|^{2}}{p^{A}}
$$

Thus

$$
\int_{\mathcal{L}^{A}} \frac{\left|\nabla_{\xi} p^{A}\right|^{2}}{p^{A}}=-\frac{d}{d t} \int_{\mathcal{L}^{A}} p^{A} \log p^{A}
$$

Combining (3.17) and (3.19) we obtain

$$
\int_{\mathcal{L}^{A}}\left|\nabla_{\xi} p^{A}\right| \leq(1-\delta)^{2} \int_{\mathcal{L}^{A}} p^{A}-\frac{C}{(1-\delta)^{2}} \frac{d}{d t} \int_{\mathcal{L}^{A}} p^{A} \log p^{A}
$$

Integrating on time and on $\mathcal{S}_{\varepsilon}^{A}$ we get

$$
\begin{aligned}
& \int_{\mathcal{S}_{\varepsilon}^{A}} \frac{1}{T} \int_{0}^{T} \frac{1}{1-\delta} \int_{\mathcal{L}^{A}}\left|\nabla_{\xi} p^{A}\right| \\
& \leq(1-\delta)\left|\mathcal{S}_{\varepsilon}^{A}\right|-\frac{C}{(1-\delta)^{3}} \frac{1}{T} \int_{\mathcal{S}_{\varepsilon}^{A}} \int_{\mathcal{S}_{\varepsilon}^{A}} p^{A}(x, d \xi, t) \log p^{A}
\end{aligned}
$$


and using the Liouville property expressed in term of the cancellation of the Kaimanovich Entropy we have

$$
\lim _{T \rightarrow+\infty} \frac{1}{T} \int_{\mathcal{S}_{\varepsilon}^{A}} \int_{\mathcal{S}_{\varepsilon}^{A}} p^{A}(x, d \xi, T) \log p^{A}(x, \xi, T)=0
$$

Choosing now $\delta_{T}$ so that we have

$$
\lim _{T \rightarrow+\infty} \frac{1}{\left(1-\delta_{T}\right)^{3}} \frac{1}{T} \int_{\mathcal{S}_{\varepsilon}^{A}} \int_{\mathcal{S}_{\varepsilon}^{A}} p^{A}(x, d \xi, T) \log p^{A}(x, \xi, T)=0
$$

and

$$
\lim _{T \rightarrow+\infty} \frac{1}{\left(1-\delta_{T}\right)^{3}} \frac{1}{T} \int_{\mathcal{S}_{\varepsilon}^{B}} \int_{\mathcal{S}_{\varepsilon}^{B}} p^{B}(x, d \xi, T) \log p^{B}(x, \xi, T)=0
$$

combining (3.16) and (3.21) we obtain

$$
\begin{aligned}
& \lim _{T \rightarrow+\infty} \int_{\mathcal{S}_{\varepsilon}^{A, A, B}} \frac{1}{T^{3}} \int_{[0, T]^{3}} \sum_{k, l, m} \delta^{k+l+m}\left|\partial \mathcal{L}_{k, \delta}^{A}(x, t)\right|\left|\mathcal{L}_{l, \delta}^{A}(y, s)\right|\left|\mathcal{L}_{m, \delta}^{B}(z, \sigma)\right| \\
& =0
\end{aligned}
$$

So extending easily this result to each term of the right-hand side of (3.15) we deduce, using (3.8) and (3.14), that, for a.e. $(x, y, z)$ in $\mathcal{S}_{\varepsilon}^{A, A, B}$, we have

$$
\begin{aligned}
& \lim _{T \rightarrow+\infty} \frac{1}{T^{3}} \int_{[0, T]^{3}} \sum_{k, l, m} \delta_{T}^{k+l+m} \operatorname{rlk}\left(\mathcal{D}_{k, \delta_{T}}^{A}(x, t) \mid \mathcal{D}_{l, \delta_{T}}^{A}(y, s), \mathcal{D}_{m, \delta_{T}}^{B}(z, \tau)\right) \\
& =\Lambda^{\star}(x, y, z)
\end{aligned}
$$

Integrating this identity on $\mathcal{S}_{\varepsilon}^{A, A, B}$ and making $\varepsilon$ tend to 0 we get the desired result and theorem 1.1 is proved.

\section{Proofs of theorems 1.2 and 1.3.}

4.1. Proof of theorem 1.2. We use the same notations as in the previous section and the outline of the proof will look very much the same. that

Take $\mathcal{S}_{\varepsilon}^{A_{i}}$ to be a saturated subset of the lamination defined by $d A_{i}$ in $\mathcal{T}_{i}$ such

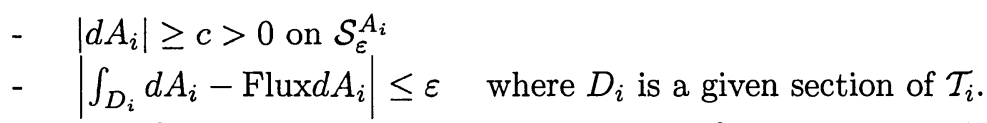

The existence of such a set is a simple consequence of the lamination hypothesis. We use the following notation $\mathcal{S}_{\varepsilon}=\mathcal{S}_{\varepsilon}^{A_{1}} \times \mathcal{S}_{\varepsilon}^{A_{2}} \times \mathcal{S}_{\varepsilon}^{A_{3}}$. We clearly have

$$
\int_{\mathcal{S}_{\varepsilon}} \frac{\left|d A_{1}\right|\left(x_{1}\right)\left|d A_{2}\right|\left(x_{2}\right)\left|d A_{3}\right|\left(x_{3}\right)}{\left|x_{1}-x_{2}\right|^{3}\left|x_{1}-x_{3}\right|^{3}} \geq 16 \pi^{4} \int_{\mathcal{S}_{\varepsilon}}\left|g^{A_{1} A_{2} A_{3}}\right|
$$

Introduce $p^{A_{i}}$ and $\tilde{p}^{A_{i}}$ like in section II. We have

$$
\int_{\mathcal{S}_{\varepsilon}}\left|g^{A_{1} A_{2} A_{3}}\right|=\int_{\mathcal{S}_{\varepsilon}} \lim _{T \rightarrow+\infty} \frac{1}{T^{3}} \int_{[0, T]^{3}} \int_{\mathcal{S}_{\varepsilon}} \tilde{p}_{A_{1}} \tilde{p}_{A_{2}} \tilde{p}_{A_{3}}\left|g^{A_{1} A_{2} A_{3}}\right|
$$


Like for (3.10) we have

$$
\begin{aligned}
& \int_{\mathcal{S}_{\varepsilon}} \tilde{p}_{A_{1}} \tilde{p}_{A_{2}} \tilde{p}_{A_{3}}\left|g^{A_{1} A_{2} A_{3}}\right| \\
& =\sum_{k_{1}, k_{2}, k_{3}} \delta^{k_{1}+k_{2}+k_{3}} \int_{\prod_{i} \mathcal{L}_{k_{i}, \delta}^{A_{i}}\left(x_{i}, t_{i}\right)}\left|g^{A_{1} A_{2} A_{3}}\right| \bigwedge_{i} d v o l_{\mathcal{L}^{A_{i}}\left(x_{i}\right)}
\end{aligned}
$$

Recall at this step that | | still denotes the norm for the canonical metric $g_{\mathbb{R}^{4}}$ on $\mathbb{R}^{4}$ but that $d v o l_{\mathcal{L}^{A_{i}\left(x_{i}\right)}}$ is the volume form for the metric induced on the leaf by $g^{A_{i}}=g_{\mathbb{R}^{4}} /\left|d A_{i}\right|$. Arguing like for establishing (3.12) we get

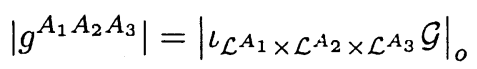

where $\mathcal{G}$ is the Gauss form introduced in proposition 2.1 and ||$_{0}$ is the scalar product for the metric on the leaves induced by $g^{A_{i}}$. Applying the definition of the Gauss-form we have

$$
\iota_{\mathcal{L}^{A_{1}} \times \mathcal{L}^{A_{2}} \times \mathcal{L}^{A_{3}}} \mathcal{G}=V^{*}(\Omega(X) \wedge \Omega(Y))
$$

where $V: \mathcal{L}^{A_{1}} \times \mathcal{L}^{A_{2}} \times \mathcal{L}^{A_{3}} \longrightarrow S^{3} \times S^{3}$ given by (1.14) and $\Omega$ is the renormalized volume form on $S^{3}$ so that $\int_{S^{3}} \Omega=1$. We then have

$$
\begin{aligned}
& \int_{\prod_{i} \mathcal{L}_{k_{i}, \delta}^{A_{i}\left(x_{i}, t_{i}\right)}}\left|g^{A_{1} A_{2} A_{3}}\right| \bigwedge_{i} d v o l_{\mathcal{L}^{A_{i}}\left(x_{i}\right)} \\
& =\int_{\prod_{i} \mathcal{L}_{k_{i}, \delta}^{A_{i}}\left(x_{i}, t_{i}\right)}\left|V^{*}(\Omega \wedge \Omega)\right|_{o} \bigwedge_{i} d v o l_{\mathcal{L}^{A_{i}}\left(x_{i}\right)}
\end{aligned}
$$

Arguing exactly like in the previous section we can, here also, replace the $\mathcal{L}_{k_{i}, \delta}^{A_{i}}$ by the $\mathcal{D}_{k_{i}, \delta}^{A_{i}}$. Indeed this require the adding of small caps that we can choose to be contained respectively in $\mathcal{T}_{1}, \mathcal{T}_{2}$ and $\mathcal{T}_{3}$. Since the supports of the three laminations are disjoint we can ensure that $\left|V^{*}(\Omega \wedge \Omega)\right|_{o}$ is uniformly bounded for a triple of points in $\mathcal{T}_{1} \times \mathcal{T}_{2} \times \mathcal{T}_{3}$ where we choosed $g^{A_{i}}$ to be the standard metric $g_{\mathbb{R}^{4}}$ on each additional cap $C_{\partial \mathcal{L}_{k_{i}, \delta}^{A_{i}}\left(x_{i}, \delta\right)}$ (it has no importance as long as the chosen metric is bounded from above and bellow relative to the standard one). Then we need to choose a good $\delta_{T}$ depending on $T$ exactly like in the previous section in order to ensure

$$
\lim _{T \rightarrow+\infty} \frac{1}{\left(1-\delta_{T}\right)^{3}} \frac{1}{T} \int_{\mathcal{S}_{\varepsilon}^{A_{i}}} \int_{\mathcal{S}_{\varepsilon}^{A_{i}}} p^{A_{i}}(x, d \xi, T) \log p^{A_{i}}(x, \xi, T)=0
$$

Like above the following choice of $\delta_{T}$ ensures

$$
\begin{aligned}
& \int_{\mathcal{S}_{\varepsilon}} \lim _{T \rightarrow+\infty} \frac{1}{T^{3}} \int_{[0, T]^{3}} \sum_{k_{1}, k_{2}, k_{3}} \delta^{k_{1}+k_{2}+k_{3}} \int_{\prod_{i} \mathcal{L}_{k_{i}, \delta}^{A_{i}}\left(x_{i}, t_{i}\right)}\left|V^{*}(\Omega \wedge \Omega)\right|_{o} \bigwedge_{i} d v o l_{\mathcal{L}^{A_{i}}} \\
& =\int_{\mathcal{S}_{\varepsilon}} \lim _{T \rightarrow+\infty} \frac{1}{T^{3}} \int_{[0, T]^{3}} \sum_{k_{1}, k_{2}, k_{3}} \delta^{k_{1}+k_{2}+k_{3}} \int_{\prod_{i} \mathcal{D}_{k_{i}, \delta}^{A_{i}}\left(x_{i}, t_{i}\right)}\left|V^{*}(\Omega \wedge \Omega)\right|_{o} \bigwedge_{i} d v o l_{\mathcal{D}^{A_{i}}}
\end{aligned}
$$


Using the Coarea formula of Federer, Since $\left|S^{3}\right|^{2} \Omega(X) \wedge \Omega(Y)$ is the standard volume form on $S^{3} \times S^{3}$ we have

$$
\begin{aligned}
& \int_{\prod_{i} \mathcal{D}_{k_{i}, \delta}^{A_{i}}\left(x_{i}, t_{i}\right)}\left|V^{*}(\Omega(X) \wedge \Omega(Y))\right|_{o} \bigwedge_{i} d \operatorname{dvol}_{\mathcal{D}^{A_{i}}} \\
& =\frac{1}{\left|S^{3}\right|^{2}} \int_{S^{3} \times S^{3}} d \sigma \operatorname{Card}\left\{\left(x_{i}\right)_{i} \in \prod_{i} \mathcal{D}^{A_{i}} \text { s.t. } V\left(\left(x_{i}\right)_{i}\right)=\sigma\right\}
\end{aligned}
$$

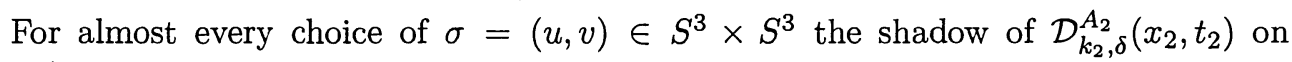
$\mathcal{D}_{k_{1}, \delta}^{A_{1}}\left(x_{1}, t_{1}\right)$, relative to $u$, is a smooth curve and the projections of both this shadow and $\mathcal{D}_{k_{3}, \delta}^{A_{3}}\left(x_{3}, t_{3}\right)$ on a plane perpendicular to $v$ are transverse and intersect each other along the set $\left\{\left(x_{1}, x_{2}, x_{3}\right) \in \prod_{i} \mathcal{D}^{A_{i}}\right.$ s.t. $\left.V\left(x_{1}, x_{2}, x_{3}\right)=\sigma\right\}$. To simplify the presentation we assume that $\Sigma_{1}, \Sigma_{2}$ and $\Sigma_{3}$ are connected. The definition of the asymptotic relative crossing number of $\Sigma_{1}, \Sigma_{2}$ and $\Sigma_{3}$ gives

$$
\begin{aligned}
& \text { Card }\left\{\left(x_{1}, x_{2}, x_{3}\right) \in \prod_{i} \mathcal{D}^{A_{i}} \text { s.t. } V\left(x_{1}, x_{2}, x_{3}\right)=\sigma\right\} \geq \\
& \operatorname{arc}\left(\Sigma_{1} \mid \Sigma_{2}, \Sigma_{3}\right)\left|\prod_{i} \operatorname{deg}\left(\mathcal{D}_{k_{i}, \delta}^{A_{i}}\left(x_{i}, t_{i}\right) ; \Sigma_{i}\right)\right|
\end{aligned}
$$

Combining (4.3), (4.5), (4.7) and (4.9) we then obtain

$$
\begin{aligned}
& \int_{\mathcal{S}_{\varepsilon}} \lim _{T \rightarrow+\infty} \frac{1}{T^{3}} \int_{[0, T]^{3}} \tilde{p}_{A_{1}} \tilde{p}_{A_{2}} \tilde{p}_{A_{3}}\left|g^{A_{1} A_{2} A_{3}}\right| \geq \operatorname{arc}\left(\Sigma_{1} \mid \Sigma_{2}, \Sigma_{3}\right) \times \\
& \int_{\mathcal{S}_{\varepsilon}} \bigwedge_{i} d x_{i} \lim _{T \rightarrow+\infty} \frac{1}{T^{3}} \int_{[0, T]^{3}} \sum_{k_{1}, k_{2}, k_{3}} \delta_{T}^{k_{1}+k_{2}+k_{3}}\left|\prod_{i} \operatorname{deg}\left(\mathcal{D}_{k_{i}, \delta}^{A_{i}}\left(x_{i}, t_{i}\right) ; \mathcal{T}_{i}\right)\right| \bigwedge_{i} d t_{i}
\end{aligned}
$$

The degree of a $\mathcal{D}^{A_{i}}$ in $\mathcal{T}_{i}$ is, by definition, the intersection number of this surface with a section $D_{i}$ of $\mathcal{T}_{i}$. So if $\omega_{i}$ is a 2 -form in $\mathcal{T}_{i}$ Poincaré dual of $D_{i}$ we have

$$
\operatorname{deg}\left(\mathcal{D}_{k_{i}, \delta_{T}}^{A_{i}}\left(x_{i}, t_{i}\right) ; \mathcal{T}_{i}\right)=\int_{\mathcal{D}_{k_{i}, \delta_{T}}^{A_{i}}\left(x_{i}, t_{i}\right)} \omega_{i}
$$

Let decompose $\int_{\mathcal{D}_{k_{i}, \delta_{T}}^{A_{i}}\left(x_{i}, t_{i}\right)} \omega_{i}=\int_{\mathcal{L}_{k_{i}, \delta_{T}}^{A_{i}}\left(x_{i}, t_{i}\right)} \omega_{i}+\int_{C_{\mathcal{L}_{k_{i}, \delta_{T}}^{A_{i}}\left(x_{i}, t_{i}\right)}} \omega_{i}, \quad$ since $\left|C_{\mathcal{L}_{k_{i}, \delta_{T}}^{A_{i}}\left(x_{i}, t_{i}\right)}\right| \leq c\left|\mathcal{L}_{k_{i}, \delta_{T}}^{A_{i}}\left(x_{i}, t_{i}\right)\right|$ we get, using (3.16), (3.21), (3.22) combined with (4.10)

$$
\begin{aligned}
& \int_{\mathcal{S}_{\varepsilon}} \lim _{T \rightarrow+\infty} \frac{1}{T^{3}} \int_{[0, T]^{3}} \tilde{p}_{A_{1}} \tilde{p}_{A_{2}} \tilde{p}_{A_{3}}\left|g^{A_{1} A_{2} A_{3}}\right| \geq \operatorname{arc}\left(\Sigma_{1} \mid \Sigma_{2}, \Sigma_{3}\right) \times \\
& \int_{\mathcal{S}_{\varepsilon}} \bigwedge_{i} d x_{i} \lim _{T \rightarrow+\infty} \frac{1}{T^{3}} \int_{[0, T]^{3}} \prod_{i}\left|\int_{\mathcal{L}^{A_{i}\left(x_{i}\right)}} \tilde{p}^{A_{i}}\left(x_{i}, \xi_{i}, t_{i}\right) \omega_{i}\right|
\end{aligned}
$$

It is not difficult to transpose the arguments of section II in order to replace $\tilde{p}^{A_{i}}$ by $p^{A_{i}}$ in the previous inequality. We have $p^{A_{i}}\left(x_{i}, \xi_{i}, t_{i}\right) d v l_{\mathcal{L}^{A_{i}}}=p^{A_{i}}\left(x_{i}, d \xi_{i}, t_{i}\right)$ and 
$\iota_{\mathcal{L}^{A_{i}\left(x_{i}\right)}}^{*} \omega_{i}=*\left(\omega_{i} \wedge \frac{d A_{i}}{\left|d A_{i}\right|}\right) d v \operatorname{vol}_{\mathbb{R}^{4}}$ where $*$ denotes the Hodge operator in $\mathbb{R}^{4}$. Using now the fact that on $\mathcal{L}^{A_{i}}\left|d A_{i}\right| d v o l_{g^{A_{i}}}=d v \operatorname{dvo}_{\mathbb{R}^{4}}$ we finally obtain

$$
\begin{aligned}
& \int_{\mathcal{S}_{\varepsilon}} \lim _{T \rightarrow+\infty} \frac{1}{T^{3}} \int_{[0, T]^{3}} \tilde{p}_{A_{1}} \tilde{p}_{A_{2}} \tilde{p}_{A_{3}}\left|g^{A_{1} A_{2} A_{3}}\right| \geq \operatorname{arc}\left(\Sigma_{1} \mid \Sigma_{2}, \Sigma_{3}\right) \times \\
& \int_{\mathcal{S}_{\varepsilon}} \prod_{i} d x_{i} \lim _{T \rightarrow+\infty} \frac{1}{T^{3}} \int_{[0, T]^{3}} \prod_{i}\left|\int_{ \pm_{\varepsilon}^{A_{i}}} p^{A_{i}} *\left(\omega_{i} \wedge d A_{i}\right)\right|
\end{aligned}
$$

Using again the ergodic theorem 2.1 for the right-hand-side of (4.12) and combining this inequality with (4.1) we have proved theorem 1.2.

4.2. Proof of theorem 1.3.. The proof of this theorem is strongly related to the proof of theorem 4.1 in [8]. Let $\Sigma_{1}^{\prime}, \Sigma_{2}^{\prime} \Sigma_{3}^{\prime}$ be 3 surfaces respectively in $\mathcal{T}_{1}, \mathcal{T}_{2}$ and $\mathcal{T}_{3}$ such that $\operatorname{deg}\left(\Sigma_{i}^{\prime} ; \mathcal{T}_{i}\right) \neq 0$. Let $u$ be a generic vector in $S^{3}$ such that the projections of $\Sigma_{1}^{\prime}$ and $\Sigma_{2}^{\prime}$ on a 3-plane perpendicular to $u$ are transverse to each other. Let

$$
S_{u}=\left\{x \in \Sigma_{1}^{\prime} \quad \text { such that } \exists y \in \Sigma_{2}^{\prime} \quad u=\frac{x-y}{|x-y|}\right\}
$$

From the definition of $\operatorname{deg}\left(\Sigma_{i}^{\prime} ; \mathcal{T}_{i}\right)$ we have, since $\mathcal{T}_{i}$ retracts on $\Sigma_{i}$,

$$
\operatorname{deg}\left(\Sigma_{i}^{\prime} ; \mathcal{T}_{i}\right)\left[\Sigma_{i}\right]=\left[\Sigma_{i}^{\prime}\right] \quad \text { in } H_{2}\left(\mathcal{T}_{i}\right) \simeq H_{2}\left(\Sigma_{i}\right)
$$

So that

$$
\left[S_{u}\right]=\operatorname{deg}\left(\Sigma_{1}^{\prime} ; \mathcal{T}_{1}\right) \operatorname{deg}\left(\Sigma_{2}^{\prime} ; \mathcal{T}_{2}\right) \sigma_{1,2} \quad \text { in } H_{1}\left(\mathcal{T}_{1}\right) \simeq H_{1}\left(\Sigma_{1}\right)
$$

Consider now $v$, a generic vector in $S^{3}$, chosen so that the half cylinder $\mathcal{C}=$ $\left\{S_{u}+t v \quad t \in \mathbb{R}_{+}\right\}$is transverse to $\partial \mathcal{T}_{3}$. For $t$ sufficiently large $\left(t \geq t_{0}\right), S_{u}+t v$ does not cross $\mathcal{T}_{3}$ anymore and we can immerse 2-disks in $\mathbb{R}^{4} \backslash \mathcal{T}_{3}$ to close $\mathcal{C}$ and to make it as a union of immersed disks transverse to $\partial \mathcal{T}_{3}$. We denote by $\mathcal{D}=\left(\mathcal{D}_{i}\right)_{i \in I}$ this family of disks $\left(\mathcal{D}_{i}=p_{i}\left(D^{2}\right)\right)$. Let $m_{i}$ be the number of component of $p_{i}^{-1}\left(\mathcal{T}_{3}\right)$ that represent a non trivial element in $H_{2}\left(\mathcal{T}_{3} ; \partial \mathcal{T}_{3}\right)$. Since $\operatorname{deg}\left(\Sigma_{i}^{\prime} ; \mathcal{T}_{i}\right)$ is the intersection with an oriented section of $\mathcal{T}_{3}$ generating $H_{2}\left(\mathcal{T}_{3}, \partial \mathcal{T}_{3}\right)$ we have

$$
\operatorname{rc}\left(\Sigma_{1}^{\prime} \mid \Sigma_{2}^{\prime}, \Sigma_{3}^{\prime}\right) \geq \sum_{i \in I} m_{i}\left|\operatorname{deg}\left(\Sigma_{3}^{\prime} ; \mathcal{T}_{3}\right)\right|
$$

We claim that

$$
m=\sum_{i \in I} m_{i} \geq\left\|\sigma_{1,2,3}\right\|\left|\operatorname{deg}\left(\Sigma_{1}^{\prime} ; \mathcal{T}_{1}\right)\right|\left|\operatorname{deg}\left(\Sigma_{2}^{\prime} ; \mathcal{T}_{2}\right)\right|
$$

This inequality is proved exactly following the proof of lemma 4.2 in [8]. Since $\pi_{1}\left(\mathcal{T}_{3} ; \partial \mathcal{T}_{3}\right)=0$, we can, following lemma 4.3 in [8], homotope the $p_{i}$ relative to the $\partial D^{2}$ 's to make it as a $m_{i}$ clean extension of the components of $S_{u}$. It is performed without increasing the number of essential components of the inverse image of $\mathcal{T}_{3}$ by $p_{i}$. So that, if $\bar{p}_{i}$ is the $m_{i}$-clean extension, $p_{i}^{-1}\left(\mathcal{T}_{3}\right)$ is made of $m_{i}$ disjoint disks in $D^{2}$ that represent non trivial elements of $H_{2}\left(\mathcal{T}_{3} ; \partial \mathcal{T}_{3}\right)$. Let $E_{i}$ be this union of disks in $D^{2}$ and $F_{i}=D^{2} \backslash E_{i}$. Then

$$
\sum_{i \in I}\left[p_{i}\left(F_{i}\right)\right]=\operatorname{deg}\left(\Sigma_{1}^{\prime} ; \mathcal{T}_{1}\right) \operatorname{deg}\left(\Sigma_{2}^{\prime} ; \mathcal{T}_{2}\right) \sigma_{1,2,3} \quad \text { in } H_{2}\left(\mathbb{R}^{4} \backslash \mathcal{T}_{3} ; \partial \mathcal{T}_{3} \cup \partial \mathcal{T}_{1}\right)
$$

We have $m_{i}-1=\left|\chi\left(F_{i}\right)\right|$ then $\sum_{i \in I} m_{i} \geq \sum_{i \in I}\left|\chi\left(F_{i}\right)\right|$ Thus we get (4.14) and theorem 1.3 is proved. 
5. An example where $\operatorname{rlk}\left(\Sigma_{1} \mid \Sigma_{2}, \Sigma_{3}\right)=0$ and $\operatorname{arc}\left(\Sigma_{1} \mid \Sigma_{2}, \Sigma_{3}\right)>0$. We give an example bellow (see figure 2) where

$$
\operatorname{rlk}\left(\Sigma_{1} \mid \Sigma_{2}, \Sigma_{3}\right)=0 \quad \text { and } \quad \operatorname{arc}\left(\Sigma_{1} \mid \Sigma_{2}, \Sigma_{3}\right)>0
$$

A short description of the link: (We mainly rely on figure 2) We take $\Sigma_{1}, \Sigma_{2}$ and $\Sigma_{3}$ so that $\Sigma_{1} \simeq T^{2}, \Sigma_{2} \simeq T^{2}$ and $\Sigma_{3}=\hat{\Sigma}_{3} \cup \tilde{\Sigma_{3}}$ where $\hat{\Sigma}_{3}$ and $\tilde{\Sigma}_{3}$ are two disjoint torii. We slice $\mathbb{R}^{4}$ by hyperplanes $H_{t}$ perpendicular to a fixed direction $e$ for $-1 \leq t \leq 1$. Let $\Sigma=\cup \Sigma_{i}, H_{t} \cap \Sigma$ is singular at exactly 8 dates $-t_{4}=-1<-t_{3}<-t_{2}<-t_{1}<$ $0<t_{1}<t_{2}<t_{3}<t_{4}=+1$ : Slicing increasingly in time we have

- for $t<-t_{4}=-1 H_{t} \cap S=\emptyset$

- for $-1<t<-t_{3} H_{t} \cap S$ is made of 4 unlinked circles.

- at $t=-t_{3} \tilde{\Sigma}_{3}$ splits into two components as shown in figure 2.

- at $t=-t_{2}$ both $\Sigma_{1}$ and $\Sigma_{2}$ split into two components as shown in figure 2 .

- at $t=-t_{1} \hat{\Sigma}_{3}$ split into 2 components as shown in figure 2 .

- $\Sigma$ is exactly symmetric relative to $H_{0}$ except that between $t=0$ and $t=t_{1}$ the left component of $\Sigma_{2}$ on figure 2 rotates exactly one time around the left component of $\hat{\Sigma}_{3}$ so that a rigid disk bounding this component of $\Sigma_{2}$ will intersect $\Sigma_{1}$ and $\tilde{S}_{3}$ along respectively the generator of $H_{1}\left(\Sigma_{1}\right)$ and the generator of $H_{1}\left(\tilde{\Sigma}_{3}\right)$ given by the left components of $\Sigma_{1}$ and $\tilde{\Sigma}_{3}$ on figure 2 (for $H_{t} \cap \Sigma-t_{1}<t<t_{1}$ ).

The class $\sigma_{1,2}$ in $H_{1}\left(\Sigma_{1}\right)$ defined in the last part of section I and obtained from the intersection of $\Sigma_{1}$ with any manifold bounding $\Sigma_{2}$ has for representative the left component on figure 2 of $\Sigma_{1} \cap H_{t}$ for $-t_{1}<t<t_{1}$. It is clear from the figure that there exists a disk bounding this component and intersecting $\Sigma_{3}$ (either $\hat{S}_{3}$ or $\tilde{S}_{3}$ ) at exactly two points with opposite intersection numbers so that

$$
\operatorname{rlk}\left(\Sigma_{1} \mid \Sigma_{2}, \Sigma_{3}\right)=0
$$

We prove now that $\operatorname{arc}\left(\Sigma_{1} \mid \Sigma_{2}, \Sigma_{3}\right)=2$. From the previous remark we have $\operatorname{arc}\left(\Sigma_{1} \mid \Sigma_{2}, \Sigma_{3}\right) \leq 2$. So we have to prove that $\operatorname{arc}\left(\Sigma_{1} \mid \Sigma_{2}, \Sigma_{3}\right) \geq 2$. If $\Sigma_{1}^{\prime}, \Sigma_{2}^{\prime}$ and $\Sigma_{3}^{\prime}=\hat{\Sigma}_{3}^{\prime} \cup \tilde{\Sigma}_{3}^{\prime}$ are three surfaces in $\mathcal{T}_{1}, \mathcal{T}_{2}$ and $\mathcal{T}_{3}=\hat{\mathcal{T}}_{3} \cup \tilde{\mathcal{T}}_{3}$ with degrees $d_{1}=\operatorname{deg}\left(\Sigma_{1}^{\prime} ; \mathcal{T}_{1}\right)$, $d_{2}=\operatorname{deg}\left(\Sigma_{2}^{\prime} ; \mathcal{T}_{2}\right)$ and $\left(\tilde{d}_{3}, \hat{d}_{3}\right)=\left(\operatorname{deg}\left(\hat{\Sigma}_{3}^{\prime} ; \hat{\mathcal{T}}_{3}\right), \operatorname{deg}\left(\tilde{\Sigma}_{3}^{\prime} ; \tilde{\mathcal{T}}_{3}\right)\right)$, then for obvious homological reasons (see the previous section) any generic 3-manifold bounding $\Sigma_{2}^{\prime}$ intersects $\Sigma_{1}^{\prime}$ along a 1-manifold homologous to $d_{1} d_{2} \sigma_{1,2}$ in $H_{1}\left(\mathcal{T}_{1}\right) \simeq H_{1}\left(\Sigma_{1}\right)$. Let $\Gamma$ be one of the connected components of this manifold. Observe from figure 2 that there exists a class $[\tau]$ in $H_{1}\left(\Sigma_{1}\right)$ which admits a representative $\tau$ whose intersection number with $\sigma_{1,2}$ is +1 , and which is bounded by a disk $\delta$ in $\mathbb{R}^{4}$ that does not intersect $\mathcal{T}_{3}$. Both $\sigma_{1,2}$ and $\tau$ generate $H_{1}\left(\mathcal{T}_{1}\right)$. If $\Gamma=d \sigma_{1,2}+\nu[\tau]$ and if $\Delta$ is any disk bounding $\Gamma$ and intersecting $\Sigma_{3}^{\prime}$ transversally it suffices then to prove that

$$
\frac{\operatorname{Card}\left(\Delta \cap \hat{\Sigma}_{3}^{\prime}\right)}{d \hat{d}_{3}}+\frac{\operatorname{Card}\left(\Delta \cap \tilde{\Sigma}_{3}^{\prime}\right)}{d \tilde{d}_{3}} \geq 2
$$

Without changing $\Delta \cap \mathcal{T}_{3}$ we can modify it in the following way : we make a small surgery by adding $\nu$ times the disk $\delta$ to $\Delta$ so that the boundary of the new disk obtained $\tilde{\Gamma}=\partial \tilde{\Delta}$ is homologous to $d \sigma_{1,2}$ we can then add to it a 2-dimensional annulus contained in $\mathcal{T}_{1}$ so that the final disk bounds $d \sigma$ where $\sigma$ is any curve generating $\sigma_{1,2}$. Thus we replace $\Gamma$ by $d$ times the curve $\sigma_{0}$ given in figure 2 by the left component of $H_{t} \cap \Sigma_{1}$ and $\Delta$ is now an immersed disk $f_{*} D^{2}\left(f:\left(D^{2} ; \partial\right) \rightarrow\left(\mathbb{R}^{4} ; \sigma_{0}\right)\right)$ bounding $d \sigma_{0}$ and we can always assume that it intersects $\mathcal{T}_{3}$ transversally and that $f: \partial D^{2} \rightarrow$ 

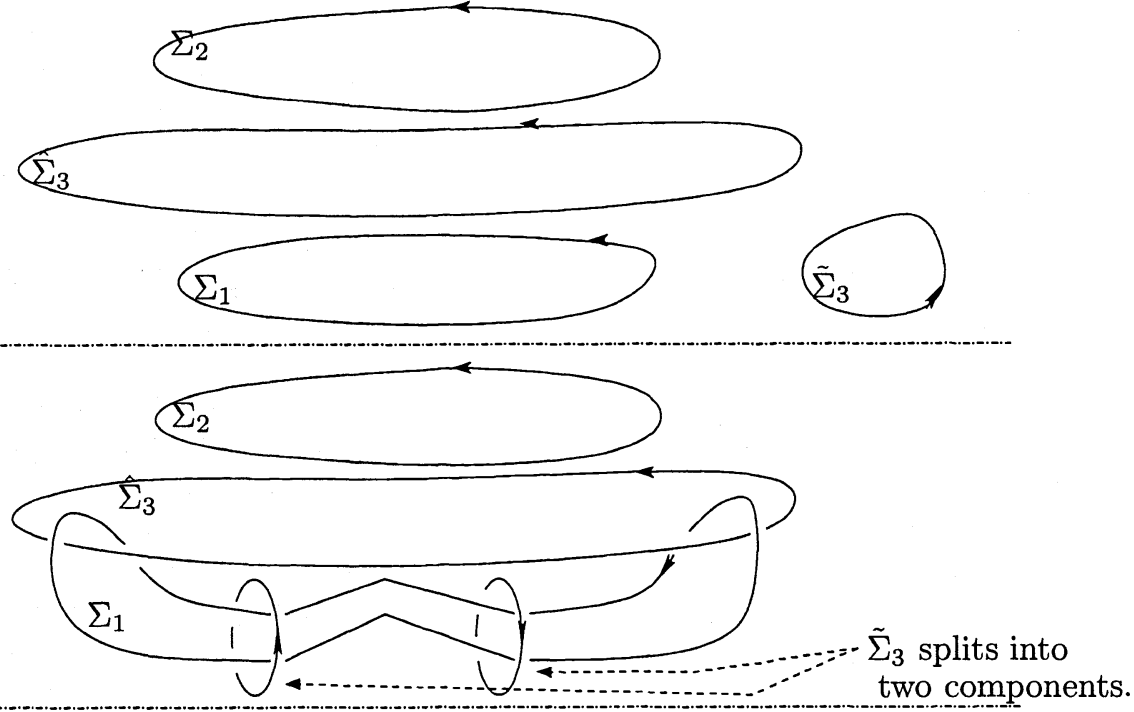

$\Sigma_{2}$ splits
into two com
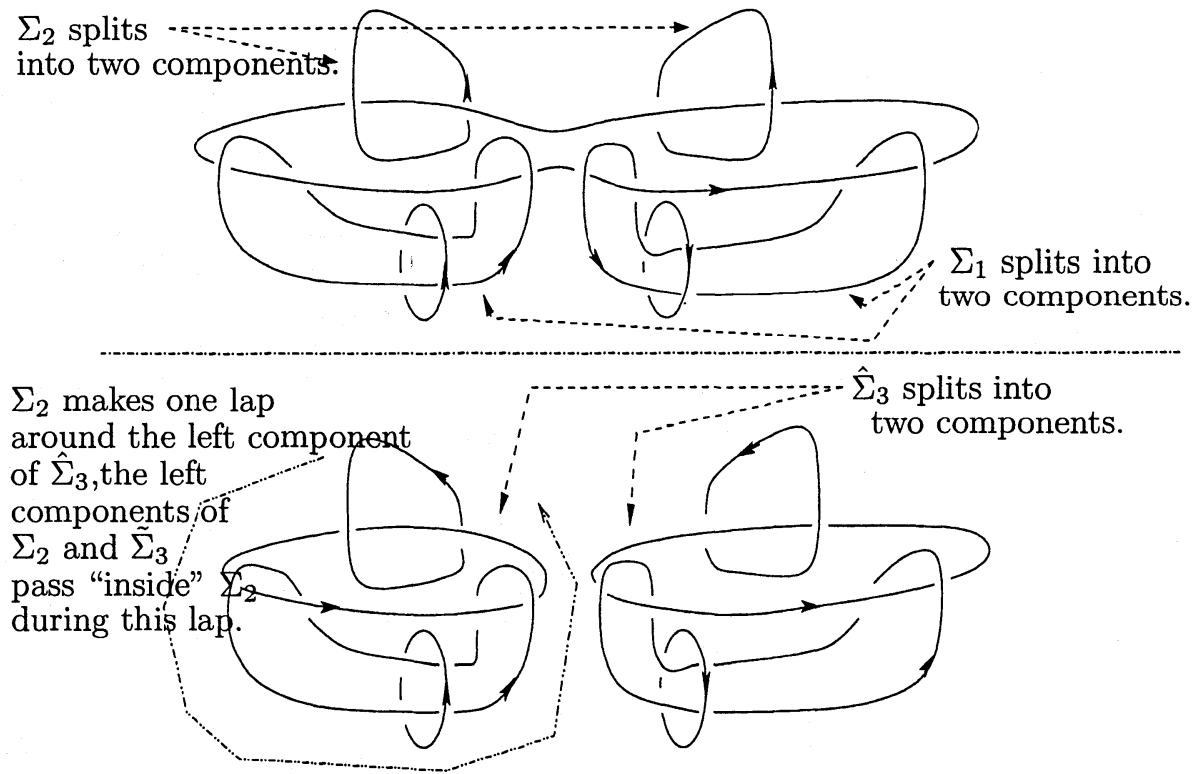

Fig. 5.1. An example where $\operatorname{rlk}\left(\Sigma_{1} \mid \Sigma_{2}, \Sigma_{3}\right)=0$ and $\operatorname{arc}\left(\Sigma_{1} \mid \Sigma_{2}, \Sigma_{3}\right)>0$

$\sigma_{0} \simeq S^{1}$ is monotonic. Let $m$ be the number of essential components of $f^{-1}\left(\tilde{\mathcal{T}}_{3}\right):$ the components whose image by $f$ are non 0 in $\pi_{2}\left(\tilde{\mathcal{T}}_{3} ; \partial \tilde{\mathcal{T}}_{3}\right)$ (has a non zero intersection number with $\tilde{S}_{3}$ ). Arguing like in [8] (lemma 4.2 and 4.3) we can deform $\Delta$ without increasing it's number of intersection with $\Sigma_{3}^{\prime}$ such that every components of $f^{-1}\left(\mathcal{T}_{3}\right)$ is a disk whose image by $f$ is essential. Let $N$ be a 3 -manifold in $\mathbb{R}^{4} \backslash \hat{\mathcal{T}}_{3}$ bounding $\tilde{\Sigma}_{3}$ such that $N \cap H_{t}$ is made of embedded disks bounding the components of $\tilde{\Sigma}_{3} \cap H_{t}$. It can be chosen so that $\sigma_{0}$ and $N$ intersect transversally at two points $p_{1}$ and $p_{2}$ we can also choose $N$ so that $\Delta$ and $N \backslash \mathcal{T}_{3}$ intersects along smooth oriented curves which are closed or starting from $p_{1}$ arriving at $\partial \tilde{\mathcal{T}}_{3}$ or $p_{2}$ or starting from $\partial \tilde{T}_{3}$ arriving at $p_{2}$. Observe that $N$ is chosen such that $N$ and $\mathcal{T}_{3}$ are separable (There exists an isotopy 
of $\mathbb{R}^{4}$ sending $N$ and $\mathcal{T}_{3}$ into opposite sides of an hyperplane in $\mathbb{R}^{4}$ ). Observe also that since $\partial N=\tilde{S}_{3}$, the intersection number of $N \cap \partial \mathcal{T}_{3}$ with an oriented section of the tubular neighborhood $\tilde{T}_{3} \simeq D^{2} \times T^{2}$ is +1 and then the difference between the number of curves of $f^{-1}\left(N \backslash \tilde{\mathcal{T}}_{3}\right)$ coming from $f^{-1}\left(p_{1}\right)$ to a component of $f^{-1}\left(\tilde{\mathcal{T}}_{3}\right)$ and the number of curves of $f^{-1}\left(N \backslash \tilde{\mathcal{T}}_{3}\right)$ leaving this component to $f^{-1}\left(p_{2}\right)$ is equal to the intersection number of the image by $f$ of this component with $\tilde{\Sigma}_{3}$. Since $\tilde{\mathcal{T}}_{3}$ deforms smoothly to $\tilde{\Sigma}_{3}$ in $\mathbb{R}^{4} \backslash \hat{T}_{3} \cup \mathcal{T}_{1}$ we may always choose $N$ so that $\partial \tilde{\mathcal{T}}_{3} \backslash N$ is diffeomorphic to $[0,1] \times \tilde{S}_{3} \simeq[0,1] \times T^{2}$. Then any connected curve on $\partial \mathcal{T}_{3} \backslash N$ whose two ends intersect $N \cap \partial \tilde{\mathcal{T}}_{3}$ with total intersection number being equal to zero can be smoothly deform in $\partial \tilde{\mathcal{T}}_{3}$, keeping it's ends fixed, to a curve in $N \cap \partial \tilde{\mathcal{T}}_{3}$. Then we deduce that we can homotope $f$ in $\mathbb{R}^{4} \backslash \hat{T}_{3}$, keeping $f\left(\partial D^{2}\right)$ fixed, keeping the number of essential components of $f^{-1}\left(\tilde{\mathcal{T}}_{3}\right)$ and their intersection number with $\widetilde{S}_{3}$ fixed, such that the number of curves of $f^{-1}\left(N \backslash \tilde{\mathcal{T}}_{3}\right)$ intersecting a component of $f^{-1}\left(\tilde{\mathcal{T}}_{3}\right)$ is equal to the intersection number between this component with $\tilde{\Sigma}_{3}$ (at this stage we take into account the sign of the intersection number, so that if it is positive we only have curves arriving from $f^{-1}\left(p_{1}\right)$ and if it is negative curves leaving for $f^{-1}\left(p_{2}\right)$ ). Since $f: \partial D^{2} \rightarrow \sigma$ is monotonic $f^{-1}\left(p_{1}\right)$ is made of exactly $d$ points alternated with $f^{-1}\left(p_{2}\right)$ which is also made of exactly $d$ points. Let $\left(\gamma_{i}\right)_{i \in I}$ to be the union of the connected curves among the one realizing $f^{-1}\left(N \backslash \tilde{\mathcal{T}}_{3}\right)$ that connect $f^{-1}\left(p_{1}\right)$ and $f^{-1}\left(p_{2}\right)$. Let $C$ be a connected component of $D^{2} \backslash \cup_{i} \gamma_{i} . f(\partial C)$ defines a class in $H_{1}\left(\mathbb{R}^{4} \backslash \hat{\mathcal{T}}_{3} ; \tilde{\mathcal{T}}_{3} \cup N\right) \simeq H_{1}\left(\mathbb{R}^{4} \backslash \hat{\mathcal{T}}_{3}\right)=\mathbb{Z}$ since $\tilde{\mathcal{T}}_{3} \cup N$ is contractible to a point in $\mathbb{R}^{4} \backslash \hat{\mathcal{T}}_{3}$. The intersection number of $C$ with $\hat{S}_{3}$ gives the class in $\mathbb{Z}$. Let $n$ be this number. Let $q$ be the algebraic number of oriented arcs in $\partial D^{2} \cap \partial C$ joining a point of $f^{-1}\left(p_{1}\right)$ and a point of $f^{-1}\left(p_{2}\right)$ : the arc oriented by $\partial D^{2}$ is counted positively if it goes from a point of $f^{-1}\left(p_{1}\right)$ to a point of $f^{-1}\left(p_{2}\right)$ and negatively in the opposite case; in the first case it counts as +1 as a contribution to $H_{1}\left(\mathbb{R}^{4} \backslash \hat{\mathcal{T}}_{3} ; \tilde{\mathcal{T}}_{3} \cup N\right)$ in the other case it counts as -1 . The difference between $q$ and the absolute number of arcs in $\partial D^{2} \cap \partial C$ joining points of $f^{-1}\left(p_{1}\right)$ and $f^{-1}\left(p_{2}\right)$ is given by the number of arc of $f^{-1}\left(N \backslash \tilde{\mathcal{T}}_{3}\right)$ in $C$ joining points of $f^{-1}\left(\left\{p_{1}\right\} \cup\left\{p_{2}\right\}\right)$ and components of $f^{-1}\left(\tilde{\mathcal{T}}_{3}\right)$. Collecting all the informations above we easily get (5.1).

Acknowledgments. The author is very gratefuhl to Robert Azencott and Laurent Younes for stimulating discussions on diffusion processes.

\section{REFERENCES}

[1] V.I. ARnold, The asymptotic Hopf invariant and its applications, Sel. Math. Sov., 5 (1986), pp. 327-345.

[2] V.I. ARnold And B.A. Khesin, Topological Methods in Hydrodynamics, Springer, AMS 125, (1999).

[3] R. BotT AND L.W. Tu, Differential forms in algebraic topology, Springer, GTM 82, (1982).

[4] I. Chavel, Eigenvalues in Riemannian Geometry, Academic Press, (1984).

[5] A. Connes, Non Commutative Geometry, Academic Press, Inc, San Diego, CA, (1994).

[6] A. Dold, Lectures on Algebraic Topology, Springer, (1980).

[7] M.H. FREEDMAN AND Z.X. HE, Links of tori and the energy of incompressible flows, Topology, 30 (1990), pp. 283-287.

[8] M.H. FReEdMAN AND Z.X. HE, Divergence-free fields : Energy and assymptotic crossing number, Ann. Math., II Ser 134, No 1 (1991), pp. 189-229.

[9] L. GARDNETT, Foliations, the ergodic theorem and Brownian motion, J. Funct. Anal., 51 (1983), pp. 285-311.

[10] U. HAMENSTÄDT, Harmonic Measures for Leafwise Elliptic Operators Along Foliations, First Europ. Congress of Math. Vol II (Paris, 1992), pp. 73-95, Progr. Math. 120, Birkhäuser, Basel, 1994. 
[11] V.A. Kaimanovich, Brownian motion and harmonic functions on covering manifolds. An entropy approach, Soviet Math. Dokl., 33 (1986), pp. 812-816.

[12] V.A. KaImanovich, Brownian motion on foliations : Entropy, invariant measures, mixing, J. Funct. Analysis, 22 (1989), pp. 326-328.

[13] B.A. KHEsin, Ergodic interpretation of integral hydrodynamic invariants, J. Geom. Phys. 9 (1992), pp. 101-110.

[14] D. KoTSCHICK AND T. VogeL, Linking Numbers of Measured Foliations, preprint (2001).

[15] H.K. MoffatT, The degree of knottedness of tangled vortex lines, J. Fluid. Mech., 35 (1969), pp. 117-129.

[16] S.P. Novikov, The analytic generalized Hopf invariant. Many-valued functionals, Russian Math. Surveys, 39:5 (1984), pp. 113-124.

[17] S.P. Novikov, Analytical theory of homotopy groups, Lecture Notes in Math., 1346, Springer (1988), pp. 99-112.

[18] J.F. Plante, Foliation with measure preserving holonomy, Ann. Math., 102 (1975), pp. 327361.

[19] T.Vogel, On the Asymptotic Linking Number, preprint (2000).

[20] P. Walters, An introduction to Ergodic Theory, Springer, GTM 79, (1982).

[21] K. YosidA, Functional analysis, Springer, Classics in Mathematics, (1995). 
